\title{
Mixing at large Schmidt number in the self-similar far field of turbulent jets
}

\author{
By WERNER J. A. DAHM† AND PAUL E. DIMOTAKIS \\ Graduate Aeronautical Laboratories, California Institute of Technology, \\ Pasadena, CA 91125, USA
}

(Received on 22 February 1988 and in revised form 12 January 1990)

We present results from an experimental investigation of turbulent transport and molecular mixing of a $S c \gg 1$ conserved scalar in the fully developed self-similar far field of a steady, axisymmetric, momentum-driven, free turbulent jet issuing into a quiescent medium. Our experiments cover the axial range from the jet exit to 350 diameters downstream, and span the range of Reynolds numbers from 1500 to 20000 . Flow visualizations of the scalar concentration field directly verify the presence of an underlying characteristic large-scale organization in the jet far field essentially consistent with a simplified conceptual picture proposed in an earlier study (Dahm \& Dimotakis 1987). High-resolution imaging measurements of successive instantaneous scalar concentration profiles in the jet support the presence of such a large-scale organization and provide details of its implications for mixing. These results also establish the proper similarity scaling for the mean concentration in the jet far field and give the scaling constant on the jet centreline as 5.4. We also present conserved scalar concentration p.d.f.s throughout the jet far field, and introduce a chemical reaction method for measuring the p.d.f.s with potentially molecular resolution. The amount of unmixed ambient fluid that reaches the jet centreline is found to decrease with increasing Reynolds number over the range investigated. The distribution of mixed fluid compositions in the concentration p.d.f. also appears to change over this range of Reynolds numbers, indicating that some aspects of large Schmidt number mixing in the jet far field have not yet become Reynolds number independent.

\section{Introduction}

Interest in the transport and mixing processes in turbulent shear flows stems mainly from their importance in a wide range of technological applications including turbulent combustion, which in the fast chemistry and low heat release limits can be determined from the transport and mixing of a conserved scalar. Classically, turbulent transport has been treated as a diffusion-like process, resulting from vortical motions whose scales are presumed small relative to the lateral extent of the flow and characteristically lacking any persistent large-scale organization. However, contrary to this classical picture, experiments over the past fifteen years have established that transport in fully turbulent plane shear layers is dominated by a characteristic large-scale and roughly periodic organization that ultimately results from the dynamics of large vortical structures (e.g. Brown \& Roshko 1971, 1974; Fiedler 1974; Winant \& Browand 1974; Dimotakis \& Brown 1976; Breidenthal

$\dagger$ Present address: Department of Aerospace Engineering, The University of Michigan, Ann Arbor, Michigan 48109-2140, USA. 
1981). This organized character of the fully developed shear layer has been found to have particularly important implications for turbulent mixing. For example, one consequence is that pure fluid from both free streams can be transported laterally across most of the layer (Konrad 1976; Mungal \& Dimotakis 1984 ; Koochesfahani \& Dimotakis 1986). A further consequence is that the range of mixed fluid compositions encountered at different lateral locations across the layer is far more uniform than what the mean concentration profile might suggest (Konrad 1976; Koochesfahani \& Dimotakis 1986). With the recognition of this organization in the shear layer and its role in turbulent transport, it has become widely presumed that some form of largescale organization may also play a significant role for transport in other turbulent free shear flows. However, in no other fully developed free shear flow have either the presence or the character of such organization been as clearly established, nor its implications for transport and mixing as well understood, as in the shear layer.

\subsection{Large scales and organized transport in turbulent jets}

In axisymmetric turbulent jets, the existence of orderly large-scale vortical structure in the near field, consisting of the first ten or so jet diameters, has been recognized for some time (e.g. Bradshaw, Ferriss \& Johnson 1964; Mollo-Christensen 1967; Becker \& Massaro 1968; Beavers \& Wilson 1970; Crow \& Champagne 1971; Yule 1973). However, this near-field organization in the jet is often viewed as transitional. Transport in the fully developed self-similar far field of turbulent jets has in the past been thought to be dominated by the classical small-scale, diffusive transport mechanism noted above. Yet the results of many investigations have indicated that some form of large-scale organization may also play an important role for transport in fully developed turbulent jets. The characteristic large-scale ramp-like features long observed in gaseous jets (e.g. Antonia, Prabhu \& Stevenson 1975; Chevray \& Tutu 1978) appear to have been early evidence of an organization in the jet far field (see Gibson, Friehe \& McConnell 1977). Tso, Kovasznay \& Hussain (1981) gave further indications for some type of large-scale organization on the basis of space and time correlations of velocity in the jet far field. In a flow visualization study, Dimotakis, Miake-Lye \& Papantoniou (1983) noted the presence of pure ambient fluid throughout the lateral extent of the jet and attributed this to entrainment by either axisymmetric or helical vortical structures in the flow. Related to this, Shlien (1987) has shown flow visualizations verifying that fluid near the jet edges is rapidly entrained deep into the jet. Dahm \& Dimotakis (1987) found that entrainment of ambient fluid to the jet centreline increased markedly during intervals typically separated by the local large-scale time of the flow, suggesting an organized entrainment mechanism closely connected with the largest local scales of the flow. Perhaps the strongest evidence of organization in the jet far field are the large-scale and quasi-periodic 'flame' length fluctuations of chemically reacting turbulent jets first attributed to organized entrainment and mixing by Dahm, Dimotakis \& Broadwell (1984), and later characterized in detail by Dahm (1985) and Dahm \& Dimotakis (1987, hereafter referred to as [I]). They found that the spectrum of length and time scales of these fluctuations were centred on the local large-scale length and time of the flow, and suggested a simplified conceptual picture for the underlying organization in the jet (see figure 7 of [I]). Mungal \& O'Neil (1989) have confirmed both the flame length fluctuations and their scaling at a much higher Reynolds number, and that the underlying large-scale organization alternates between axisymmetric and spiral modes (see also figures $2 b$ of Mungal \& Hollingsworth 1989). Papantoniou (1985) and Papantoniou \& List (1989) have confirmed that most of 
these ideas from momentum-driven turbulent jets can also be applied to buoyant turbulent jets. When these results are collected together, there is considerable evidence for a large-scale organization of transport and mixing in the fully developed far field of turbulent jets, though the details of this organization are quite different from those in the shear layer and are only poorly understood in comparison. One of the objectives of the investigation reported here is to further clarify the nature of any large-scale organization in the jet far field and its implications for mixing.

\subsection{Small scales and molecular mixing}

The classical picture of transport in turbulent flows noted above also has implications for mixing. In accordance with this diffusion-like view of the transport mechanism, the resulting turbulent fluxes of scalars are presumed to be directly proportional to the local gradients in their mean profiles through a turbulent diffusivity that is modelled as a function of the flow. In fully developed turbulent flows, this turbulent gradient transport of mass is presumed to dominate molecular diffusion, and the resulting effect of the molecular diffusivity on mixing is taken as negligible. However, again contrary to this classical view, experiments in fully turbulent shear layers (Mungal \& Dimotakis 1984; Koochesfahani \& Dimotakis 1986) have shown that the amount of molecular mixing, measured by the amount of chemical product formed in a fast chemical reaction, differs by as much as a factor of two for a gaseous layer and a liquid layer at comparable Reynolds numbers, demonstrating a definite effect of the molecular diffusivity on the degree of mixing achieved. The term mixing will be used throughout this discussion to refer specifically to molecular mixing. A second objective of this work is to contribute to the understanding of whether the indications of large-scale organization in the jet far field also imply an attendant effect of the molecular diffusivity in jet mixing.

The role of molecular diffusion in mixing can be characterized by the Schmidt number $S c \equiv \nu / D$ as the ratio of the vorticity and scalar diffusivities. The molecular transport kinetics in gaseous mixing lead to $S c$ of $O(1)$, while in liquids $S c$ is much larger. In the mixing of conserved scalars in turbulent flows, the competing effects of the local strain rate in the flow and molecular diffusion of the scalar establishes a local limiting thickness $\lambda_{D}$ giving the smallest local scale on which gradients in the conserved scalar field can be sustained by the flow. The corresponding balance between the strain rate and diffusion of vorticity imposes a similar limit $\lambda_{\nu} \sim \delta R e^{-\frac{3}{4}}$ on the smallest spatial scale of gradients in the vorticity field, where $R e \equiv u \delta / \nu$ is the local Reynolds number based on the velocity $u$ and length $\delta$ characterizing the shear at that stage in the flow. The ratio of the vorticity and scalar diffusivities then establishes the relation between these scales as $\lambda_{D} \sim \lambda_{v} S c^{-\frac{1}{2}}$, where the similarity of the two limiting processes suggests a proportionality constant of order one. Thus for mixing in gases, $\lambda_{D}$ is of the order of $\lambda_{v}$, while in water $(S c \approx 600) \lambda_{D}$ is typically about 25 times smaller under the same conditions. This also points out the relatively greater difficulty in conducting high-resolution measurements of mixing at large Schmidt numbers. Of course, these difficulties are further compounded, for both gases and liquids, by the decrease in the Kolmogorov scale with increasing Reynolds number.

The effects of these differences due to the molecular diffusivity on various measures of mixing in turbulent jets will be most clearly discerned by comparing measurements spanning a wide range of the Schmidt number. To date, however, virtually all investigations of jet mixing have been conducted in gaseous (low $S c$ ) media. A noteworthy exception are the experiments by Weddell (reported in Hottel 1953). 
They studied the large-Schmidt-number mixing between aqueous acid and base solutions and used a reactive scalar (phenolphthalein) to directly indicate the distance required for the jet fluid to completely mix with ambient fluid to any given degree. Weddell's measurements, however, provide few details of the transport and mixing processes. The same can be said for virtually all studies of mixing in gaseous jets, which have focused largely on single point measurements of the conserved scalar field (e.g. Becker, Hottel \& Williams 1967; Antonia et al. 1975; Birch et al. 1978; Pitts $\&$ Kashiwagi 1984). Moreover, few of these measurements have had the necessary spatial or temporal resolution to distinguish the full range of scales involved in the conserved scalar field. A further objective of this work is to provide detailed measurements of large Schmidt number conserved scalar mixing in turbulent jets which, over at least a limited range of Reynolds numbers, resolve essentially all scales arising in the scalar field.

\subsection{Present work}

The study reported here is an investigation of transport and large Schmidt number mixing of a conserved scalar in the fully developed, self-similar far field of a turbulent jet. The results we present are from qualitative flow visualization experiments as well as quantitative, detailed imaging measurements of the conserved scalar field in the jet. They address several specific aspects of jet mixing. First, some of these results aim to help clarify the nature of large-scale organization in the jet far field and its role in molecular mixing. Second, some of these results are aimed at establishing the proper similarity scalings for the mean and fluctuating concentrations in the jet far field. Further, these results determine the distribution of mixed fluid compositions throughout the jet in terms of the conserved scalar concentration probability density functions (p.d.f.s). They especially attempt to quantify the amount of unmixed ambient fluid in the jet and the variation in the p.d.f.s with Reynolds number. Collectively, these results are intended to provide a baseline against which future, high-resolution, detailed studies of mixing in gaseous turbulent jets can be compared to discern the role of the molecular diffusivity in mixing. However, it should be noted that several of the results presented here also apply directly to mixing in gaseous jets.

The presentation is organized as follows. Section 2 gives a brief description of the experiments and the conserved and reactive scalar techniques used. In $\$ 3$ we present results from flow visualizations of the instantaneous conserved scalar field in the jet and discuss their indications for organized mixing in turbulent jets. Section 4 describes the line imaging techniques used to obtain successive, high-resolution, axial and radial profiles of the conserved scalar field, and in $\$ 5$ we present results from these measurements and describe some of their implications for jet mixing. Further discussion of these results and conclusions are given in $\$ 6$. In addition, several concepts important to this paper and to the subject in general are developed in the Appendix.

\section{Experimental overview}

Most of the experiments described here use the mass fraction of a water-soluble laser fluorescent dye (disodium fluorescein) carried by the jet fluid as a conserved scalar to mark the local extent of molecular mixing between the jet and ambient fluids. Although such conserved scalar techniques are commonly used to measure mixing in turbulent flows, whenever the physical resolution of the measurement is incapable of distinguishing the smallest spatial or temporal scales of mixing, as is 
generally the case, then the local scalar concentration cannot be determined unambiguously. Nevertheless, even without complete resolution capabilities such conserved scalar measurements can still provide useful estimates of the local extent of mixing.

To circumvent such resolution limitations, in some of these measurements we exploited the $\mathrm{pH}$-sensitive characteristics of disodium fluorescein to use the dye as a reactive scalar capable of directly indicating if the local extent of mixing at the molecular scale had exceeded a given value. This approach was also used by Koochesfahani \& Dimotakis (1986). Additional details of the technique as it is used here are given by Dahm (1985). Briefly, by choosing aqueous acid $\left(\mathrm{H}_{2} \mathrm{SO}_{4}\right)$ and base $(\mathrm{NaOH})$ solutions as the jet and ambient fluids, the dye could be arranged to fluoresce only if its local concentration did not exceed a particular value. This reversible fluorescence transition could be made quite sharp by choosing the absolute strengths of the reactants to be sufficiently high, and could be arranged to occur at any desired value of the ambient-to-jet fluid mixture ratio, referred to here as the stoichiometric mixture ratio $\phi$, by appropriately selecting the relative reactant strengths. This acid-base neutralization reaction and the accompanying fluorescence transition occur on a nanosecond timescale. In all these experiments, the dye concentration was sufficiently weak (typically less than $10^{-6} \mathrm{M}$ ) to have no effect on the jet fluid density. Thus, for the conserved scalar experiments, the jet and ambient fluid densities were matched by matching their temperature as closely as possible, while for the reactive scalar measurements in $\$ 5.5$ this was done by dissolving a salt $\left(\mathrm{Na}_{2} \mathrm{SO}_{4}\right)$ in the ambient fluid. In all cases buoyancy effects were entirely negligible even at the farthest downstream measurement location (see Papanicolaou \& List 1988).

The experiments were conducted in water in a tank having $33 \times 33 \times 62$ in. interior dimensions. The jet fluid discharged from a $3_{4}^{\frac{3}{4}}$ in. diameter plenum with a strong axisymmetric contraction through a $\frac{1}{10}$ in. inner diameter nozzle. Beyond this smooth contraction, the nozzle had a length to diameter ratio of only 8-to-1 to give a very nearly uniform velocity profile across the jet exit. A cruciform insert eliminated any swirl in the plenum, which could lead to a significant swirl component in the jet exit velocity. The jet fluid was discharged slightly below the free surface in the tank by driving the plenum with an air stream metered through a micrometer-controlled variable-throat sonic orifice with a constant upstream pressure. An important consideration in any such non-coflowing jet mixing experiment is the recirculation and re-entrainment of jet fluid that must occur as the jet impinges on the bottom of the tank. In these experiments, the farthest downstream measurement location was sufficiently far from the bottom of the tank that no such self-contamination of the flow occurred. A related consideration is the local counterflow that must be induced in the ambient fluid by the local mass flux of the jet. For the dimensions of our facility, this induced counterflow velocity never exceeded $2.5 \%$ of the local mean centreline velocity.

Dimensional reasoning indicates that the Reynolds number is constant in the jet far field and proportional to $\left(J_{0} / \rho_{\infty}\right)^{\frac{1}{2}} / v_{\infty}$, where $J_{0}$ is the jet source momentum flux and $\rho_{\infty}$ and $v_{\infty}$ are the density and kinematic viscosity of the ambient fluid. With the jet and ambient fluid densities matched and with a circular jet source having an essentially uniform exit velocity profile, this Reynolds number is equivalent to, and usually written as, $u_{0} d_{0} / \nu_{\infty}$, where $u_{0}$ and $d_{0}$ are the jet exit velocity and diameter, respectively. The results reported here are for Reynolds numbers of 1500,5000 and 20000 , corresponding to exit velocities as high as $8 \mathrm{~m} / \mathrm{s}$. Note that although some of 
these Reynolds numbers are necessarily low to meet resolution requirements, as pointed out by Broadwell (1987) the associated Péclet numbers (ReSc) are high owing to the large Schmidt number, and as a result the smallest diffusion layer thicknesses $\left(\lambda_{D} / \delta\right) \sim R e^{-\frac{3}{4}} S c^{-\frac{1}{2}}$ are comparable with gaseous jet mixing at Reynolds numbers more than 70 times higher. Moreover, the experiments in water by Weddell (in Hottel 1953), and similar ones by Dahm et al. (1984), show that at least some aspects of large $S c$ mixing in jets, such as the flame length of chemically reacting jets, have already become Reynolds number independent by $R e \approx 3000$.

\section{Visualizations of the conserved scalar field}

In this section we present flow visualizations of conserved scalar transport and mixing in the far field of the jet using planar laser-induced fluorescence. The flow was intersected by a thin laser sheet containing the jet axis, and dye-containing fluid in the plane of the laser sheet then fluoresced with an intensity proportional, in part, to the local jet fluid concentration. Figure $1(a-c)$ shows typical results for the instantaneous conserved scalar concentration field from the jet source to 350 diameters downstream at increasing values of the Reynolds number.

Several features of particular interest can be identified from the photographs in figure 1. First, these photographs and numerous others like them directly show that the instantaneous conserved scalar concentration field in the jet is typically arranged in an ordered sequence of large regions within which the composition of the mixed fluid, as indicated by the grey shading, shows relatively little variation, in contrast to the differences in composition apparent between successive regions. Indeed, these regions are most easily identified by the abrupt jump in mixed fluid composition in going from one region to the next, though the boundaries separating them are topologically quite complex at finer scales. Photographs as well as motion films of this type show that the typical size and spacing of these regions increase linearly with downstream position, as would be expected from the self-similar scaling of the flow, and that their lateral scale typically spans the full local width $\delta(x)$ of the flow. Early indications of such regions were noted by Dimotakis, Broadwell \& Howard (1983a) and Dimotakis (1983b). Their presence is indirectly implied by the 'flame' length fluctuations observed by Dahm et al. (1984) and in [I], where a conceptual picture of this underlying organization was presented. The photographs in figure 1 directly verify the presence of such a large-scale organization in the jet and confirm the essential elements of this conceptual picture. However, while figure 7 of [I] suggests a largely axisymmetric arrangement of these regions, such as in figure $1(c)$, there are often indications of a more nearly spiral mode, such as in figure $1(b)$. Evidence of a helical structure in the jet was also seen in the flow visualizations of Dimotakis et al. $(1983 b)$, and is further suggested by the flame length fluctuations of Mungal \& O'Neil (1989). From photographs of this type, we find these regions to be consistently more recognizable at the comparatively higher Reynolds numbers, such as in figures $1(b)$ and $1(c)$. Their continued presence at very much higher Reynolds numbers is also implied by the flame length fluctuations of Mungal \& O'Neil at a Reynolds number of 76000 .

Direct visualizations such as those in figure 1 also show that unmixed ambient fluid is intertwined with the mixed fluid within these regions over a very wide range of scales. Indeed, such unmixed ambient fluid can be found throughout the far field of the jet, including the centreline of the flow. Moreover, the photographs in figure 1 suggest that the amount of unmixed ambient fluid found in the jet decreases with 
(a)

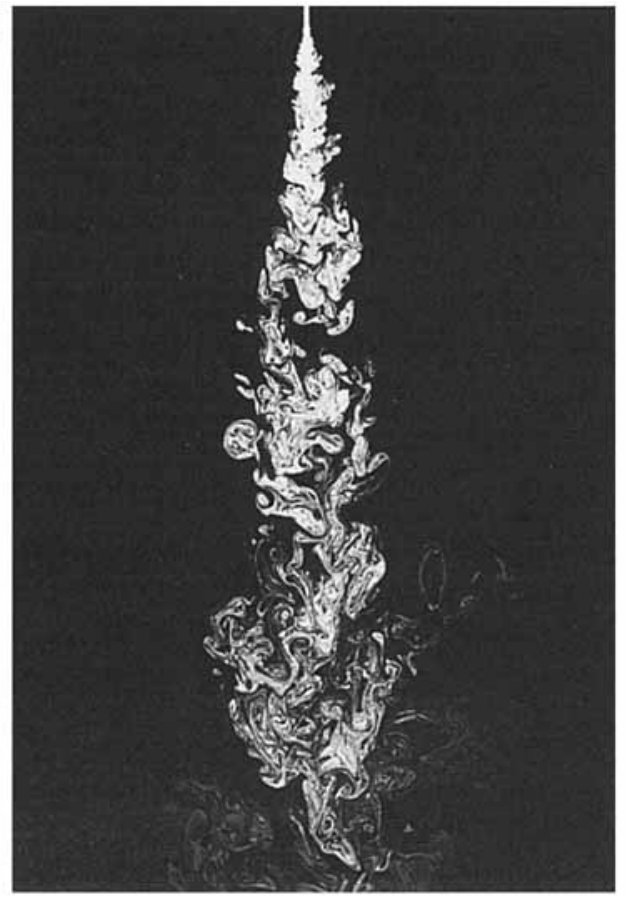

(b)

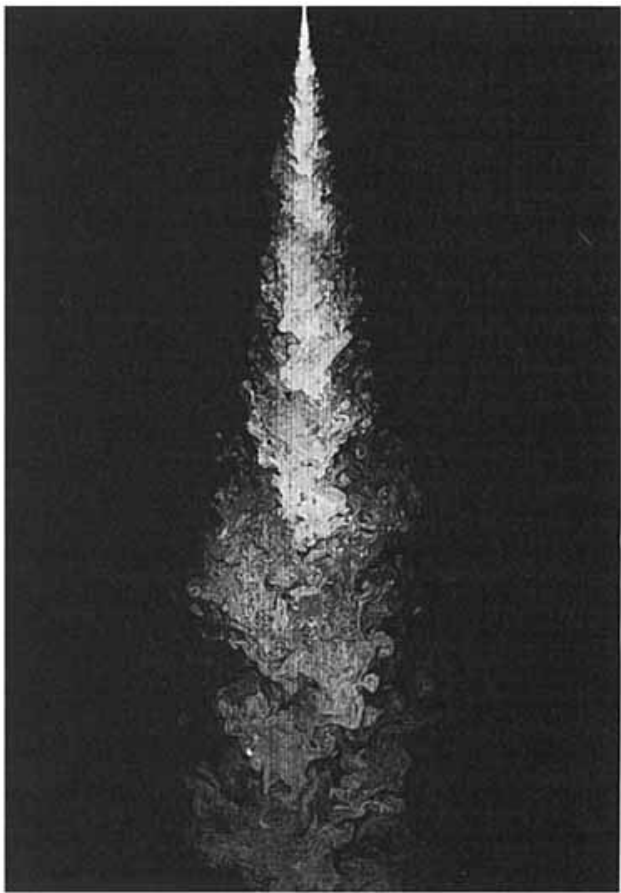

(c)

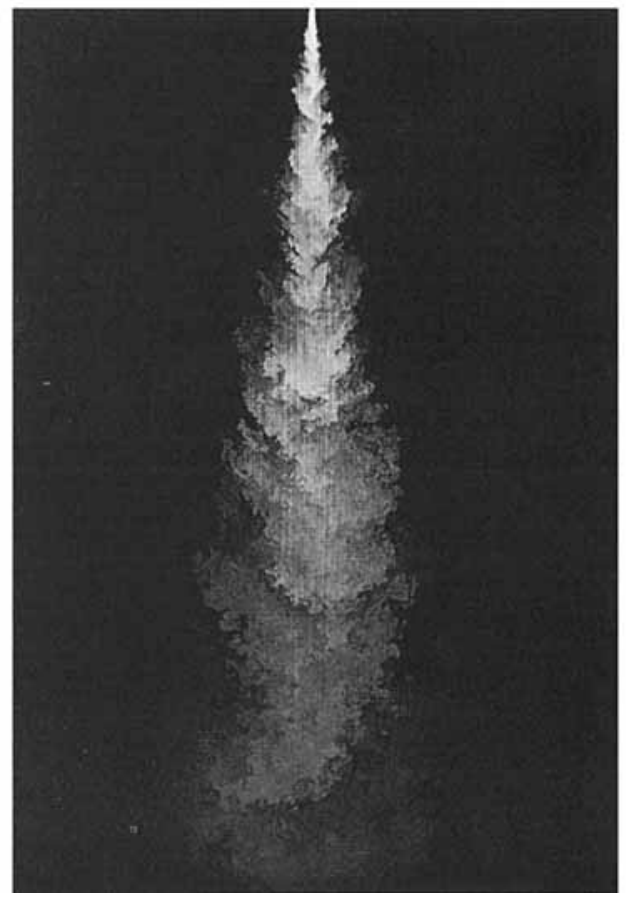

Figure 1. Planar laser-induced fluorescence photographs of the instantaneous conserved scalar concentration in the jet far field at increasing Reynolds numbers. The area shown extends 350 jet exit diameters downstream. Notice the presence of large regions within which the jet fluid concentration, as indicated by the grey shading, is approximately uniform. Notice also the presence of unmixed ambient fluid throughout the entire lateral extent of the flow in the self-similar far field. (a) $R e=1500$, (b) $R e=5000$, (c) $R e=20000$. 
increasing Reynolds number. It is important to note, however, that with increasing Reynolds number the corresponding decrease in the strain-limited molecular diffusion scale $\lambda_{D}$ renders an increasing fraction of the unmixed ambient fluid near the finest scales unresolvable by the fixed laser sheet thickness. As a consequence, at least part of the apparent decrease in the amount of unmixed fluid evident in these photographs with increasing Reynolds number may be attributable to such resolution ambiguity. This key point, namely how much pure ambient fluid reaches the jet centreline at various Reynolds numbers, is one of the issues addressed quantitatively in the following sections. It is noteworthy, however, that recent highresolution single-point measurements of conserved-scalar concentrations in gaseous (low $S c$ ) turbulent jets by Dowling (1988) and Dowling \& Dimotakis (1988) show that no pure ambient fluid reaches the centreline in the jet far field at Reynolds numbers comparable with these, demonstrating an effect of the Schmidt number on mixing in turbulent jets.

\section{Concentration profile measurement technique}

In this section we describe the line imaging technique used to measure successive, instantaneous, high-resolution profiles of the conserved scalar concentration field in the jet; results from these measurements are presented in $\S 5$. The technique used was similar in many respects to measurements of concentration in the plane shear layer by Koochesfahani \& Dimotakis (1986). Briefly, we measured successive instantaneous profiles of the conserved scalar concentration in the jet far field by imaging the laser-induced fluorescence along a line onto a linear photodiode array. To eliminate possible effects of $\mathrm{pH}$ variations in the water on the dye fluorescence characteristics, softened water fixed at $\mathrm{pH}=9$ by the addition of a small amount of $\mathrm{NaOH}$ was used for both the jet and ambient fluids. For our axial profile measurements, the beam $(514.5 \mathrm{~nm})$ from an argon ion laser was collimated and oriented coincident with and propagating up the jet centreline. The fluorescence from dye along the beam was imaged onto a 1024-element self-scanning linear photodiode array (Reticon RL-1024G). An orange filter eliminated directly scattered laser light from any particulates in the flow. Each of the 1024 photodiode array elements had a sensitive detector aperture of $25 \mu \mathrm{m} \times 26 \mu \mathrm{m}$. The imaging optics used produced an image ratio of $30: 1$, and the beam diameter varied from roughly $0.5 \mathrm{~mm}$ to about $1 \mathrm{~mm}$, thereby defining the volume imaged onto each array element. The 1024element array was clocked at a pixel rate of $256 \mathrm{kHz}$, corresponding to a scan rate of 235 scans/s (including a blanking time of 64 additional clock cycles per scan), giving a scan time of $4.3 \mathrm{~ms}$. The serial pixel output of the array was digitized through a single channel, 8-bit A/D converter and recorded directly on the computer disk. The imaged portion of the jet axis spanned the range from $0 \leqslant \chi \leqslant 300$, where $\chi \equiv\left(x / d^{*}\right)$ is the axial coordinate properly normalized in the far field by the jet source momentum diameter $d^{*}$ (see Appendix), with

$$
d^{*} \equiv \frac{2 \dot{m}_{0}}{\left(\pi \rho_{\infty} J_{0}\right)^{\frac{1}{2}}} .
$$

We also measured radial profiles of the conserved scalar concentration using essentially the same imaging technique and the same data rates. In this case, the collimated laser beam crossed the jet radially at $\chi=300$ perpendicular to the jet axis. The optics produced an image ratio of $26: 1$ and the beam diameter again varied 


\begin{tabular}{|c|c|c|c|c|}
\hline & \multicolumn{3}{|c|}{$x$} \\
\hline & & 100 & 200 & 300 \\
\hline \multirow{3}{*}{$R e$} & 1500 & $0.41 \mathrm{~mm}$ & $0.83 \mathrm{~mm}$ & $1.24 \mathrm{~mm}$ \\
\hline & 5000 & $0.17 \mathrm{~mm}$ & $0.34 \mathrm{~mm}$ & $0.50 \mathrm{~mm}$ \\
\hline & 20000 & $0.06 \mathrm{~mm}$ & $0.12 \mathrm{~mm}$ & $0.18 \mathrm{~mm}$ \\
\hline
\end{tabular}

TABLE 1. Estimates of the strain-limited molecular diffusion layer thickness $\lambda_{D}$ at various axial locations for the Reynolds numbers at which measurements were conducted. These estimates should be compared to the $(0.75 \mathrm{~mm})^{3}$ typical image volume to assess the spatial resolution of these measurements relative to the finest scales appearing in the conserved scalar field, as discussed in $\$ 4.1$.

from about $0.5 \mathrm{~mm}$ to $1 \mathrm{~mm}$, giving essentially the same pixel image volume dimensions as for the axial measurements.

\subsection{Resolution}

The relative spatial resolution of these measurements can be estimated by comparing the pixel image volume dimensions with the smallest lengthscales on which gradients can occur in the conserved scalar field. Following the discussion in $\$ 1.2$ and assuming that Kolmogorov scaling applies at these Reynolds numbers, the strain-limited molecular diffusion layer thickness could be estimated as $\left(\lambda_{D} / \delta\right) \sim R e^{-\frac{3}{4}} S c^{-\frac{1}{2}}$, where $\delta \approx 0.44 x$ is the local lateral scale of the flow. Recent measurements by Dowling (1988) suggest that the constant in this proportionality is roughly 25, and direct measurements of $\lambda_{D}$ by Dahm \& Buch (1989) appear to confirm this. With $S c \approx 600$ in these experiments, this gives the estimates of $\lambda_{D}$ at various downstream locations listed in table 1 . On the basis of these estimates, the spatial resolution of our measurements was sufficient to fully resolve the jet fluid concentration field for $x \geqslant 150$ at $R e=1500$, while at the farthest downstream measurement location the size of the pixel image volume was nearly sufficient to resolve $\lambda_{D}$ at $R e=5000$, but was roughly four times larger than $\lambda_{D}$ at $R e=20000$.

The relative temporal resolution can also be estimated by comparing the array scan time of $4.3 \mathrm{~ms}$ with the typical passage time for strain-limited molecular diffusion layer thickness $\lambda_{D} / u$, where from Chen \& Rodi $(1980) u \approx 7.0\left(J_{0} / \rho_{\infty}\right)^{\frac{1}{2}} x^{-1}$ is the local mean axial velocity on the centreline. The values for $\lambda_{D}$ in table 1 then give the estimates in table 2 for the passage time $\lambda_{D} / u$. Note that since, at the lower Reynolds numbers and at large axial distances, the array scan time was fast relative to the local timescales in table 2 , and in order to keep the amount of data collected manageable, only every 16 th scan of the array was recorded at $R e=1500$, while every 6 th scan was recorded at $R e=5000$ and every 5 th scan at $R e=20000$. With this timing arrangement, the time between successive recorded array scans was $68.8 \mathrm{~ms}, 25.8 \mathrm{~ms}$ and $21.5 \mathrm{~ms}$ respectively. Comparing these data rates with the times in table 2 shows that the resulting temporal resolution capabilities were essentially comparable with the spatial resolution at the farthest downstream measurement location. Note also that, with this timing arrangement, the data rate relative to the local large-scale flow time $\delta / u$ was the same at Reynolds numbers 1500 and 5000 , and was one-fourth this value at 20000 . Each measurement at the two lower Reynolds numbers consisted of 4000 recorded scans of the array, while at Reynolds number 


$\operatorname{Re}\left\{\begin{array}{rrrr} & \multicolumn{3}{c}{\chi} \\\right.$\cline { 2 - 4 } 1500 & 100 & 200 & \multicolumn{1}{c}{300} \\ 5000 & $11.9 \mathrm{~ms} & 47.6 \mathrm{~ms} & 107.0 \mathrm{~ms} \\ 20000 & 1.5 \mathrm{~ms} & 5.8 \mathrm{~ms} & 13.0 \mathrm{~ms} \\ & 0.1 \mathrm{~ms} & 0.5 \mathrm{~ms} & 1.2 \mathrm{~ms}\end{array}$

TABLE 2. Estimates of the strain-limited molecular diffusion layer passage time $\lambda_{D} / u$ at various axial locations for the Reynolds numbers at which measurements were conducted. These estimates are compared in $\$ 4.1$ with the $4.3 \mathrm{~ms}$ image time to assess the relative temporal resolution of these measurements.

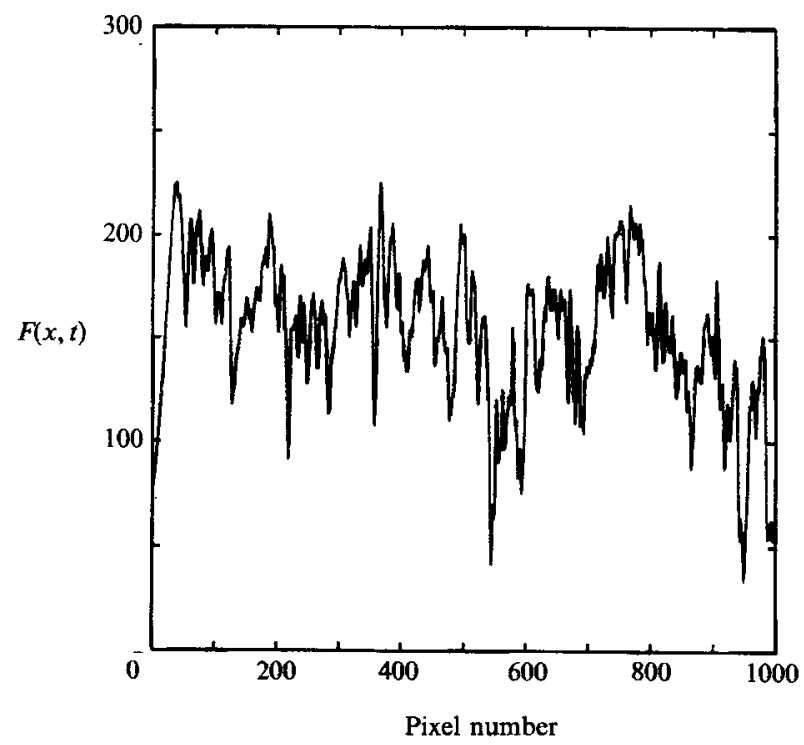

Frgure 2. A typical instantaneous fluorescence intensity profile $F(x, t)$ measured on the jet centreline over the range $0 \leqslant \chi \leqslant 300$ at $R e=5000$. Note that the typical signal level is uniform over the entire measured profile (see $\$ 4.2$ ).

20000 a total of 1000 array scans were recorded for each measurement. Each data set therefore spanned the same duration relative to the timescales of the flow, corresponding to approximately twelve large-scale flow times at $\chi=300$.

\subsection{Dynamic range}

Measurements of axial concentration profiles over this wide range of the jet centreline pose a significant dynamic range problem. Since the jet fluid concentrations from $0 \leqslant \chi \leqslant 300$ span more than two decades in magnitude, recording direct measurements of concentration to a digital resolution of 8 bits would permit distinguishing a large number of concentration levels near the jet exit, where the concentrations are high, but by $\chi \approx 140$ the concentrations would have decreased to the point where fewer than 10 digital concentration levels could be distinguished. This problem can be circumvented, however, by noting that although the typical dye concentrations along the centreline decrease owing to mixing, the intensity of the beam, which propagates in the opposite direction, decreases owing to absorption and fluorescence by the dye. This suggests that by appropriately choosing the 


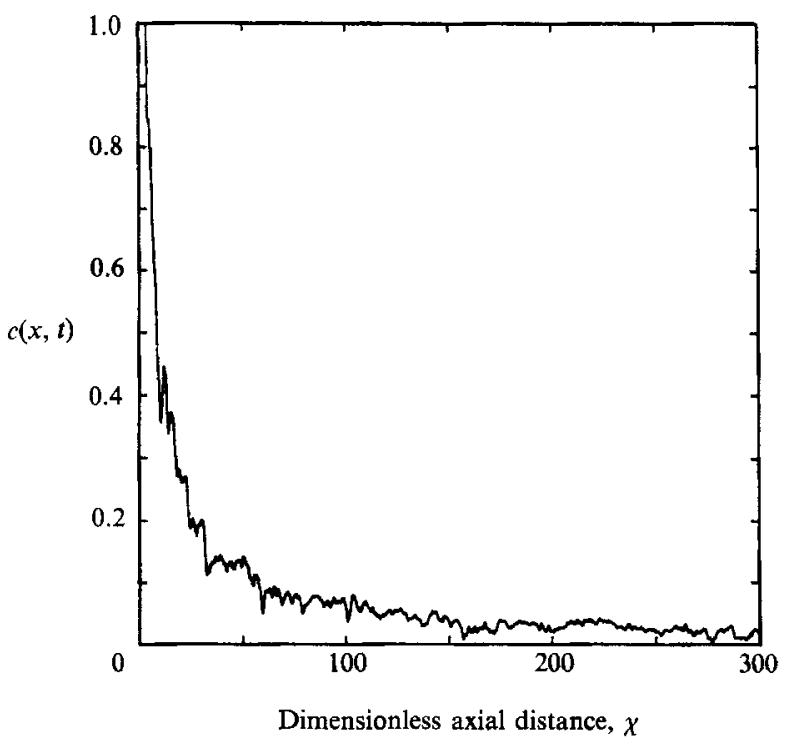

Figure 3. The instantaneous jet fluid concentration profile $c(x, t)$ obtained from the measured fluorescence intensity profile $F(x, t)$ in figure 2 (see $\$ 4.3$ ). Each measurement typically consisted of 4000 such successively measured jet fluid concentration profiles.

concentration of dye in the pure jet fluid, the attenuation of the beam could be arranged to very nearly offset the dilution of the dye, producing an axial fluorescence intensity profile that can be made very nearly uniform over the entire extent of the measurement, and thereby offering uniform dynamic range for the entire profile. Figure 2 shows a typical individual profile of the fluorescence intensity $F(x, t)$ along the jet centreline measured using this approach.

\subsection{Conversion to concentration profiles}

The measured pattern of background illumination and fixed dark noise was subtracted from each such individual axial or radial fluorescence intensity profile. Each profile was subsequently converted to the corresponding jet fluid concentration profile, $c(x, t)$, using the technique described in [I]. Briefly, an exact line attenuation integral accounted for the effects of attenuation along the beam, while the measured image transfer function accounted for the collective non-idealities in the optical system and the array. The jet exit provided the required reference point for the axial measurements, while for the radial measurements a small vessel containing a circulating solution at a known dye concentration was used as the reference. For the fluorescence profile in figure 2, the resulting jet fluid concentration profile is shown in figure 3. In the radial measurements, the resulting concentration profiles were subsequently expressed in the self-similar form $g(\eta, t)$, where $\eta \equiv r / x$, by normalizing each instantaneous profile with the local mean centreline concentration as

$$
g(\eta, t) \equiv \frac{c(x, r, t)}{\bar{c}(x, r=0)} .
$$




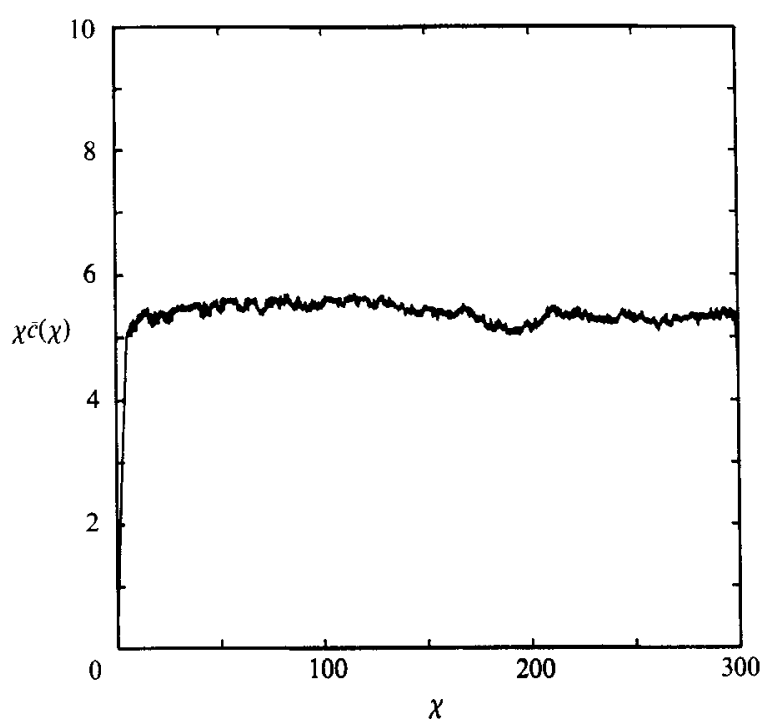

Figure 4. The profile $\chi c(\chi)$ obtained from the axial measurements at $R e=5000$ as a test of the $\chi^{-1}$ similarity demanded of the mean concentration profile $\bar{c}(\chi)$. The fact that the resulting $\chi \bar{c}(\chi)$ remains essentially constant throughout the far field verifies the flow quality attained and the imaging technique used.

\section{Concentration profile measurement results}

\subsection{Time-averaged concentrations}

The mean jet fluid concentration profiles along the centreline, $\bar{c}(\chi)$, resulting from an ensemble average of the individual axial concentration profiles measured in these experiments, are used as a partial validation of the flow quality and the imaging technique used. As demonstrated in the Appendix, self-similarity in the jet far field requires that the mean concentration profile along any ray in the jet $(\eta=$ const.) should follow a $\chi^{-1}$ scaling. Though it is popular to check the linearity of the $1 / \bar{c}(\chi)$ profile, subject to the choice of virtual origin, as a test of similarity, a more telling measure of the extent to which the required similarity scaling is satisfied by the mean profile is to examine the resulting $\chi \bar{c}(\chi)$ profile, which should then remain constant in the self-similar far field. For the $\tilde{c}(\chi)$ profile obtained from our measurements at $R e=5000$, the corresponding $\chi \bar{c}(\chi)$ profile is shown in figure 4 . Note that no virtual origin was needed for our data. The fact that this profile becomes essentially constant after a sufficiently large $\chi$ and remains at nearly this same value for the full extent of the measurement is a strong verification of the measurement and data reduction techniques as well as the similarity scaling demanded of the flow.

Profiles of $\chi \bar{c}(\chi)$ from measurements reported in other studies over the range $0 \leqslant x \leqslant 100$ are shown together with our results in figure 5 . A further advantage of the $\chi \bar{c}(\chi)$ profile is that it directly gives the numerical value of the scaling constant in the mean centreline concentration scaling. Notice that there is considerable disagreement in the value of this scaling constant among the measurements shown in figure 5, with reported values ranging from 4.3 to 5.6. Much of this disagreement is likely to be a result of variations in the validity of the approximation $d^{*} \approx\left(\rho_{0} / \rho_{\infty}\right)^{\frac{1}{2}} d_{0}$ commonly made in forming the dimensionless coordinate $\chi$, as discussed in the Appendix. The jet nozzle in our experiments was specifically designed so that this approximation 


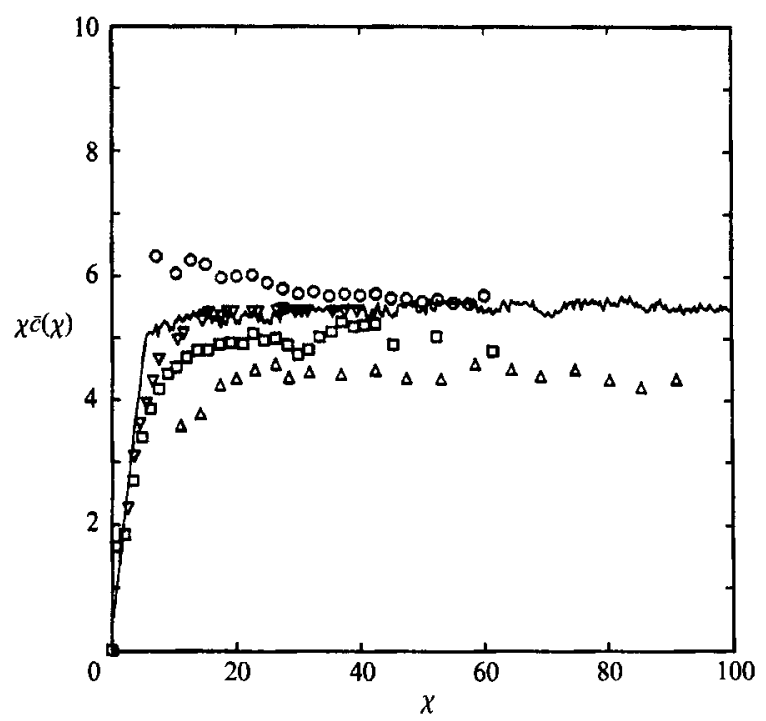

Figure 5. The profile $\chi \bar{c}(\chi)$ from figure 4 compared with results from other investigations over the range $0 \leqslant \chi \leqslant 100$, giving the scaling constant for the mean centreline concentration profile in the self-similar far field. Note the disagreement in the values of the scaling constant reported from various investigations. $\square$, Lockwood \& Moneib (1980); O, Wilson \& Danckwerts (1964); $\triangle$, Birch et al. (1978); $\nabla$, Becker et al. (1967); - - Present study.

can be used. Note that the value for the scaling constant obtained from our profile in figure 4 is

$$
\chi \bar{c}(\chi)=5.4 \pm 0.14 \text {. }
$$

This result can be compared with the upper bound of 5.70 and the approximate value of 5.15 obtained from the integral mass balance in the Appendix. The value of the scaling constant along any other ray $\eta$ is then simply $5.4 g(\eta)$, where $g(\eta)$ as defined in (2) is the mean radial concentration profile in the far field. The profile $g(\eta)$ resulting from an average of the 4000 measured instantaneous radial profiles at $R e=5000$ is shown in figure $5(a)$ of $[\mathrm{I}]$ and compares well with the mean profiles reported from other measurements.

\subsection{R.m.s. concentration fluctuations}

The axial profile of r.m.s. concentration fluctuations along the jet centreline, $c^{\prime} / \bar{c}$, obtained from the present measurements at $R e=5000$ is shown in figure 6 , together with the profiles reported from other experiments. Also shown is the r.m.s. concentration fluctuation value obtained on the centreline from our radial profile measurements at $\chi=300$ (see figure $5 b$ of $[\mathrm{I}]$ ). Note that there is considerable disagreement in the fluctuation levels reported from various investigations. It appears likely that this disagreement is primarily due to the differing levels of spatial and temporal resolution, relative to the finest scales in the concentration field, represented among the various measurements. Mean concentration measurements such as those in $\$ 5.1$ are, of course, insensitive to such resolution inadequacies. Irrespective of the lack of agreement among the various measurements shown, it is generally assumed that the concentration fluctuations $c^{\prime} / \bar{c}$ will reach a constant level in the self-similar jet far field, yet figure 6 shows that few measurements, including the present, actually report attaining such a constant value. It appears very likely that this also is in part a result of resolution inadequacies, since for any given measurement the absolute resolution is typically fixed while the spatial and temporal 


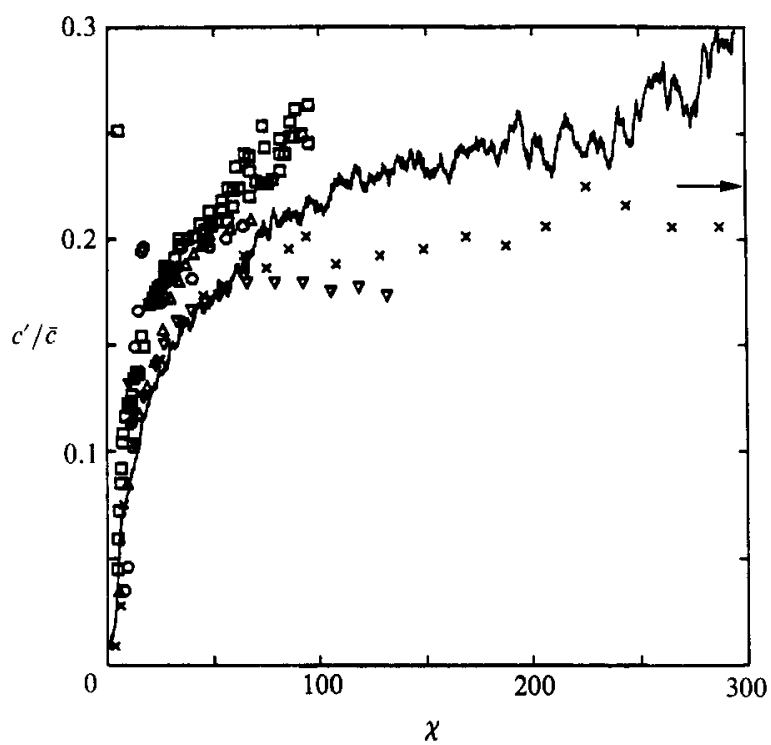

Figure 6. The profile of r.m.s. concentration fluctuations $c^{\prime} / \bar{c}$ along the jet centreline over the range $0 \leqslant \chi \leqslant 300$ obtained from the axial measurements at $R e=5000$ and from other investigations (see $\$ 5.2$ ). The value $c^{\prime} / \bar{c}=0.225$ obtained from our radial profile measurements at $\chi=300$ is also shown. $\square$, Birch et al. (1978); 0 , Becker et al. (1967); $\nabla$, Wilson \& Danckwerts (1964); $\times$, Nakamura et al. (1982); $\triangle$, Lockwood \& Moneib (1980); —_, Present axial measurements; $\longrightarrow$, Present radial measurements.

scales associated with the fluctuation intensities increase as $\chi$ and $\chi^{2}$, respectively. As a consequence, the relative resolution increases with increasing axial distance. This is certainly true for the present measurements, as is detailed in $\$ 4.1$. Note, however, that while our axial and radial profile measurements have essentially the same resolution at $\chi=300$, the radial measurements give the fluctuation level as $c^{\prime} / \bar{c}=0.225$, while the axial profile results suggest a higher level. It is likely that this is due to small beam steering effects in the axial measurements which produce spurious intensity fluctuations at the reference point beyond those removed by the attenuation integral (see $\$ 4.3$ ). The nature of the radial profile measurements renders them much less susceptible such beam steering effects, and as a result the value 0.225 for the fluctuation level obtained from the radial measurements should be quite accurate.

\subsection{Concentration time traces}

The measured time variation in the jet fluid concentration at $R e=5000$ for several different radial locations across the jet at a fixed axial location $(\chi=300)$ is shown in figure $7(a-d)$. Time is shown normalized with the local large-scale time of the flow, $\delta / u$. As discussed in $\$ 4.1$, the spatial and temporal resolution at these conditions were both nearly sufficient to resolve $\lambda_{D}$.

One noteworthy result from concentration traces such as those in figure 7 is that, when mixed fluid is present at a point near the jet edges, its jet fluid concentration is not nearly as low as the mean profile might suggest. For example, in figure $7(a)$, although the mean concentration at this radial location is only about 0.2 , the data show that when mixed fluid is present its jet fluid concentration is typically much higher. Indeed, in comparing the traces in figure $7(a-d)$, there is relatively little variation in the mixed fluid composition along the radial direction. Thus the mean concentration at any radial position is more directly indicative of the fraction of time 

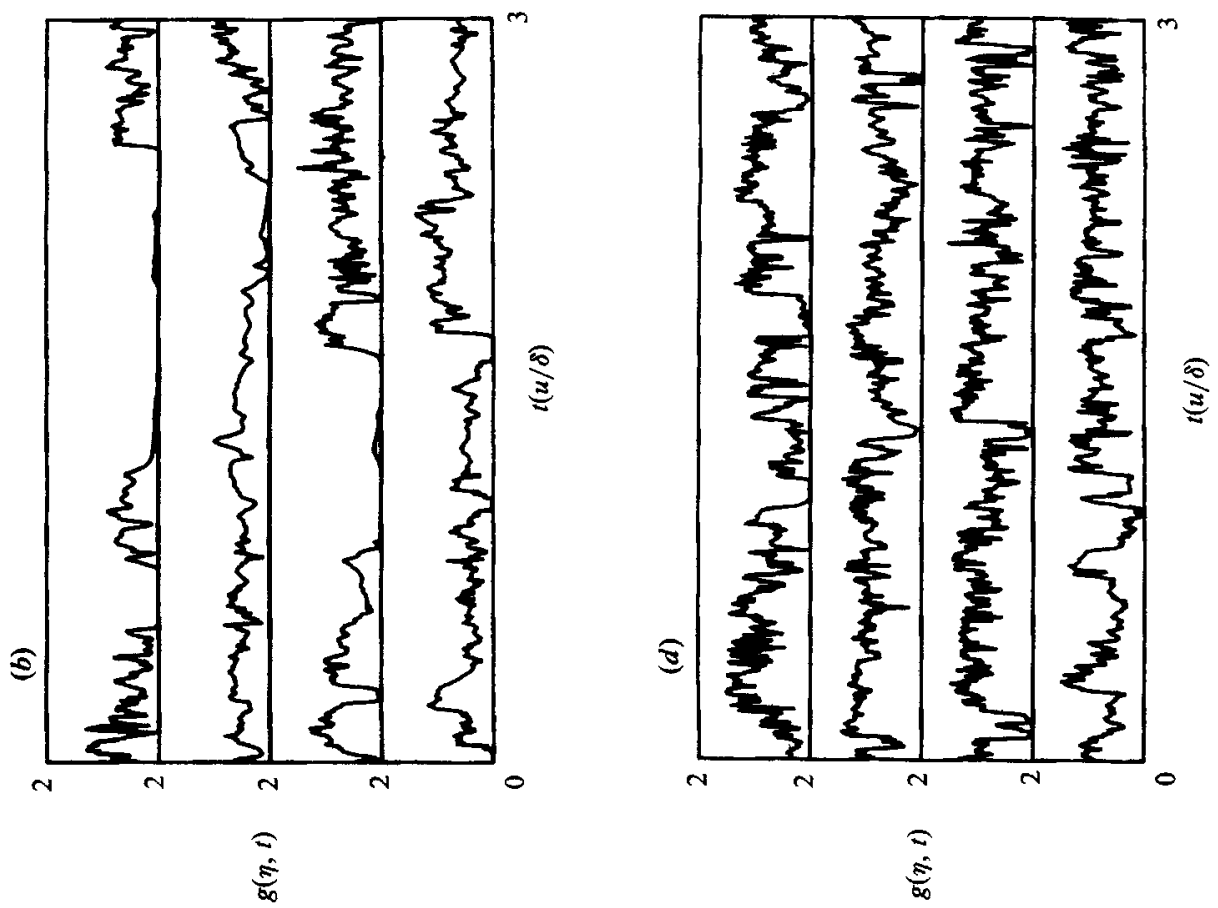

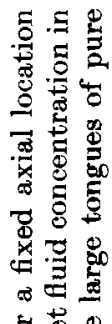

- 5.

ङ

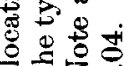

表它 11

का

용

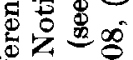

声

苟

焉峦

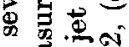

邑

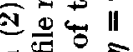

ฐ
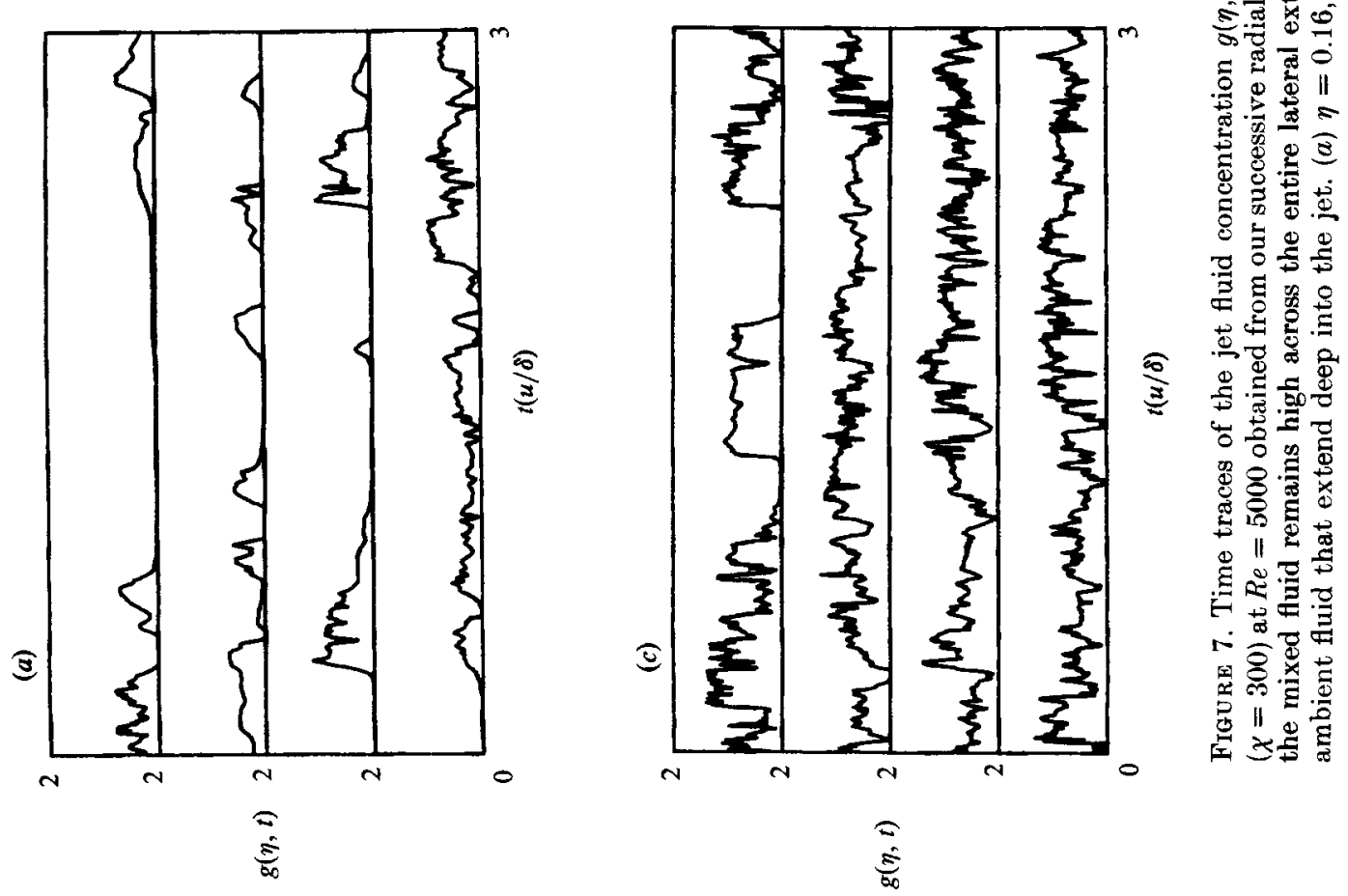

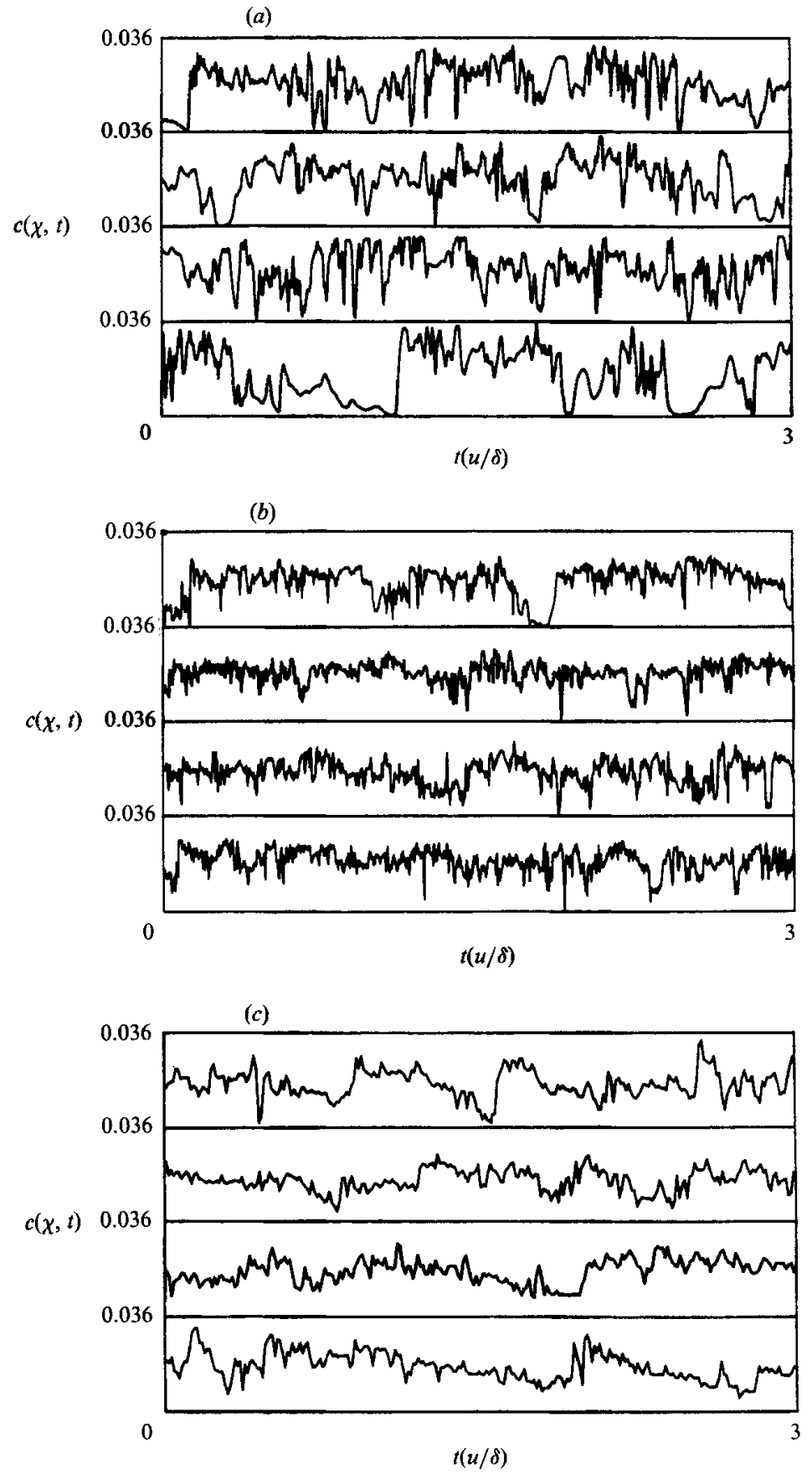

Figure 8. Time traces of the jet fluid concentration $c(\chi, t)$ on the jet centreline in the self-similar far field $(\chi=300)$ at increasing values of the Reynolds number. Notice that the amount of pure ambient fluid reaching the jet centreline appears to decrease with increasing Reynolds number (see $\S 5.3$ ). (a) $R e=1500,(b) R e=5000,(c) R e=20000$. 
that the point is occupied by mixed fluid than it is indicative of the typical jet fluid concentration in the mixed fluid at that point. While this is somewhat related to the classical notion of intermittency, the main emphasis here is on the observation that the jet fluid concentration in the mixed fluid remains high across the entire jet radius. This is borne out from a somewhat different perspective by instantaneous radial profiles of concentration across the jet, where essentially no consistent lateral gradient in the mixed fluid composition is seen within the large regions noted in $\$ 3$ (e.g. figures 6 and 7 of [I]). Again the similarity with the turbulent shear layer is noteworthy (e.g. Fiedler 1974; Mungal \& Dimotakis 1984), where as pointed out in $\S 1$ the large spanwise vortices act to prevent sustained gradients in the mixed-fluid composition across the flow.

Figures $8(a-c)$ shows time traces of the jet fluid concentration on the jet centreline $(\chi=300, \eta=0$ ) with increasing Reynolds number. A further noteworthy result from these traces, as well as those in figure 7 , is that they do not show the characteristic large-scale ramp-like features previously reported in gaseous $(S c \approx 1)$ turbulent jets. Such ramps are evident, for example, in figure $24(b)$ and 25 of Antonia et al. (1975), figure 18 of Chevray \& Tutu (1978), and figure 15 of Pitts \& Kashiwagi (1984). No indication of such ramps can be seen in figure 7 or in either of figures $8(a)$ or $8(b)$. In figure $8(c)$, at the highest Reynolds number, a few such features spanning roughly one local large-scale time $(\delta / u)$ can be identified, but there is no clear or consistent pattern of such ramps in our data, despite the regions evident in figure 1 and the evidence in [I] of a slight decrease in concentration from the front to the rear of these regions. While we cannot offer a direct explanation for the absence of such ramps in the $S c \gg 1$ time traces in figures 7 and 8 , we note that this situation appears similar to an apparent phenomenon found in turbulent shear layers. Specifically, Mungal \& Dimotakis (1984) report traces of product concentration (temperature) in a gaseous layer which exhibit large ramp-like features much like those found in jets, while product concentration traces in a liquid layer by Koochesfahani \& Dimotakis (1986) do not exhibit such ramps. Broadwell \& Mungal (1986) have given an explanation for this Schmidt-number effect in shear layers, based on mixing between freshly entrained fluid and previously mixed fluid (see also the discussion in Mungal \& Frieler 1985). In their explanation, the transport of pure entrained fluid deep into the flow by the large-scale vortices in the shear layer plays a key role in the formation of such ramps at low $S c$ and in their absence at high Sc. By comparing the traces at the various radial locations in figure 7 , evidence can be seen for similar transport of pure ambient fluid deep into the jet. Indeed, large tongues of unmixed ambient fluid extending well into the flow can be identified in these data, suggesting that a similar mechanism might be responsible for the absence of such ramps in our data.

The data in figure 7 also show that the amount of unmixed ambient fluid found within the jet decreases as the centreline is approached. The time traces in figure 8 further suggest that the amount of unmixed ambient fluid reaching the jet centreline decreases with increasing Reynolds number. At $R e=1500$, the data in figure $8(a)$ show pure ambient fluid reaching the jet centreline in the far field, yet considerably less such fluid is seen in the data at $R e=5000$ in figure $8(b)$, and at $R e=20000$ in figure $8(c)$ no unmixed ambient fluid appears to reach as far as the jet centreline. While this seems consistent with the observations in figure 1, it must be kept in mind that these measurements as well as the visualizations in figure 1 suffer increasing resolution difficulties with increasing Reynolds number. To address this issue, an experiment was conducted to determine how the probability of detecting unmixed ambient fluid on the jet centreline, $\bar{p}_{0}(0)$, varied with measurement resolution, and 
(a)

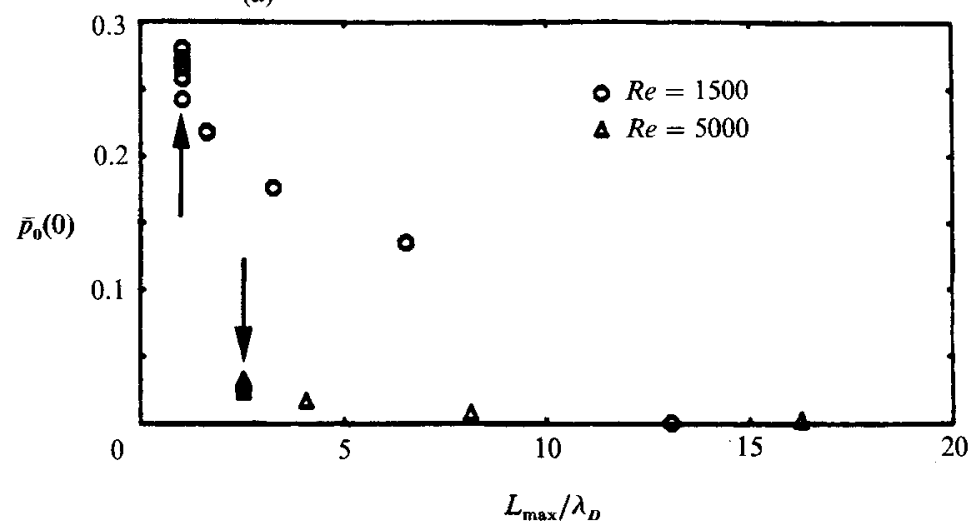

(b)

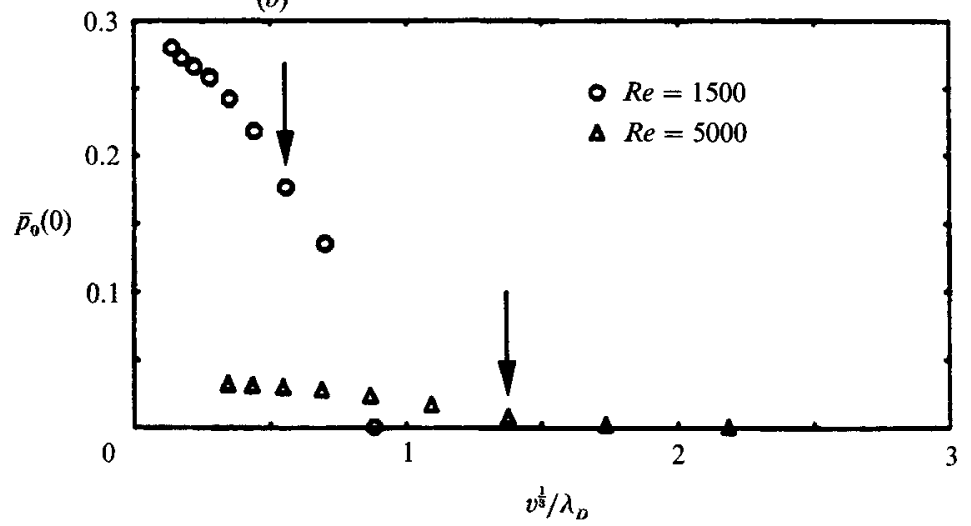

FIGURE 9. Effects of measurement resolution on the amount of unmixed ambient fluid detected at the jet centreline (see §5.3). The arrows at each Reynolds number indicate the resolution at which the bulk of the measurements were conducted. (a) Resolution based on the maximum dimension $L$ of the image volume. (b) Resolution based on the image volume $v$.

how the amount of pure ambient fluid reaching the centreline varied with Reynolds number. In this case, the image ratio was reduced so that the volume in the flow imaged onto each pixel was approximately 75 times smaller than before. The probability $\bar{p}_{0}(0)$ was then determined by discriminating between mixed and unmixed fluid from the measured fluorescence intensities, with the threshold between these set by the peak dark noise in the array. The effect of resolution could then be assessed by purposely degrading the effective resolution by averaging the measured fluorescence intensity over two or more adjacent pixels before applying the mixed/unmixed criterion. The result of this procedure is shown in figure 9, where the resolution is quantified both in terms of the effective image volume $v$, and its maximum dimension $L_{\max }$, relative to $\lambda_{D}$. Note that both the image volume and its maximum dimension can affect the resolution attained and thereby influence the value of $\bar{p}_{0}(0)$ obtained. In particular, note that $\bar{p}_{0}(0)$ increases even when $L_{\max } / \lambda_{D}$ is held fixed and $v^{\frac{1}{3}} / \lambda_{D}$ is decreased. If we can extrapolate in figure $9(b)$ to infinitely fine resolution, these data suggest that the fraction of the jet centreline is the far field occupied by pure ambient fluid decreases from nearly $30 \%$ to less than $4 \%$ as the Reynolds number is increased from 1500 to $\mathbf{5 0 0 0}$. Although even these values for $\bar{p}_{0}(0)$ must still be viewed as approximations, we can conclude that, even when 


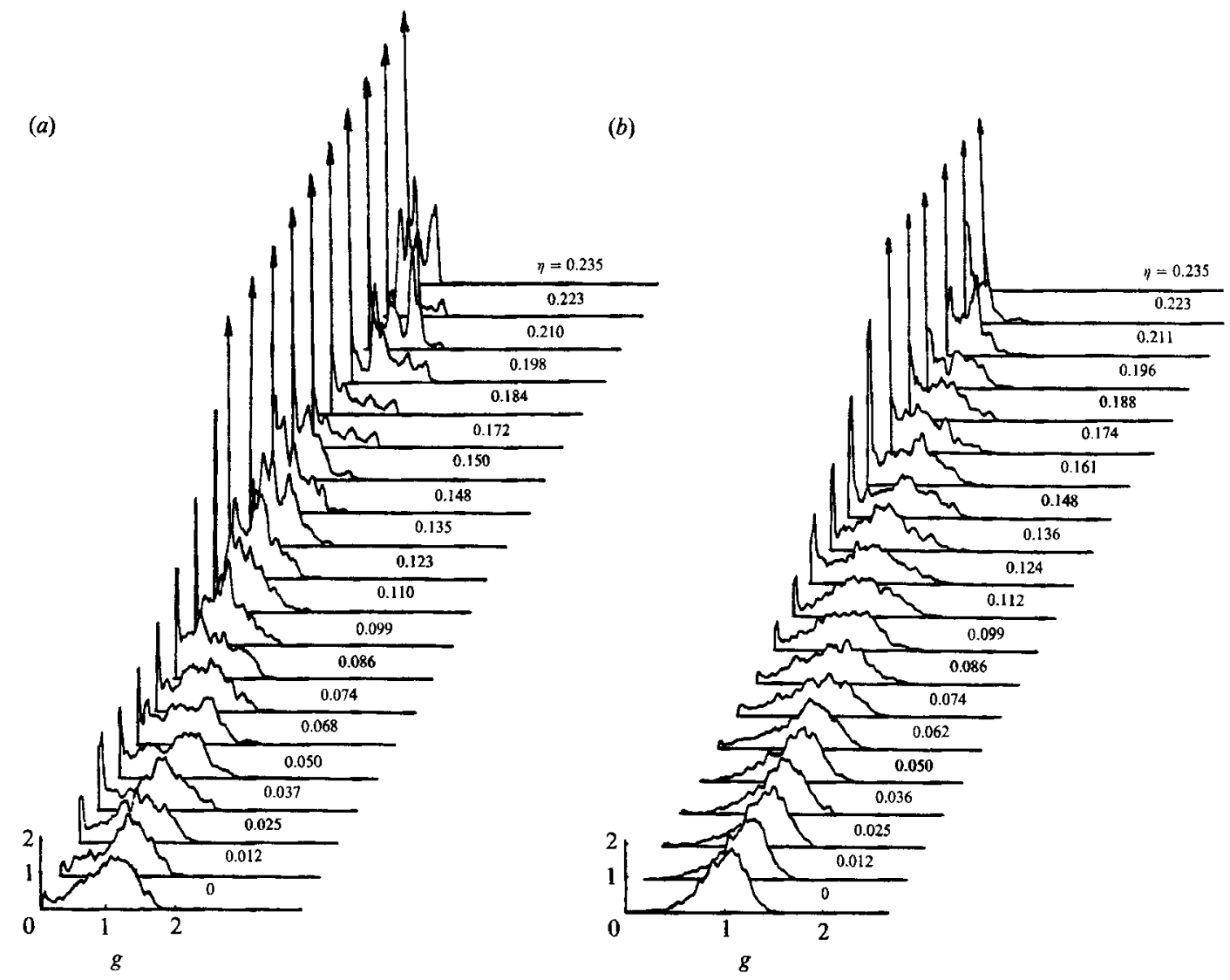

FIGURE 10. P.d.f.s of the jet fluid concentration $g(\eta, t) \equiv c / \bar{c}$ along the radial direction in the selfsimilar far field of the jet $(\chi=300)$ obtained from the radial profile measurements. Note how the amount of unmixed ambient fluid decreases as the jet centreline is approached and with increasing Reynolds number. (a) $R e=1500$, (b) $R e=5000$.

resolution effects are taken into account, less unmixed ambient fluid reaches the jet centreline with increasing Reynolds number.

\subsection{Concentration probability density functions (p.d.f.s)}

The local concentration probability density function (p.d.f.) gives the distribution of conserved scalar values found at that point in the flow, quantifying the timeindependent transport and mixing properties of the jet. Indeed, local statistics of the jet concentration field which do not depend directly on the concentration history can be derived from the p.d.f.s. The significance of the p.d.f. for characterizing transport and mixing points to the importance associated with accurately determining its form throughout the jet far field. Direct measurements of the conserved scalar concentration p.d.f.s at Reynolds numbers 1500 and 5000 , given in terms of the radial similarity concentration $g$, are shown in figure $\mathbf{1 0}$ for a fixed axial location $(\chi=300)$ at several different radial locations $\eta$ covering the entire radial extent of the jet. Together with the mean concentration scaling $5.4 g(\eta)$ from $\$ 5.1$, these p.d.f.s give the distribution of jet fluid concentrations throughout the entire far field of the jet. Note that, even at the higher Reynolds number, for this axial location the 
(a)
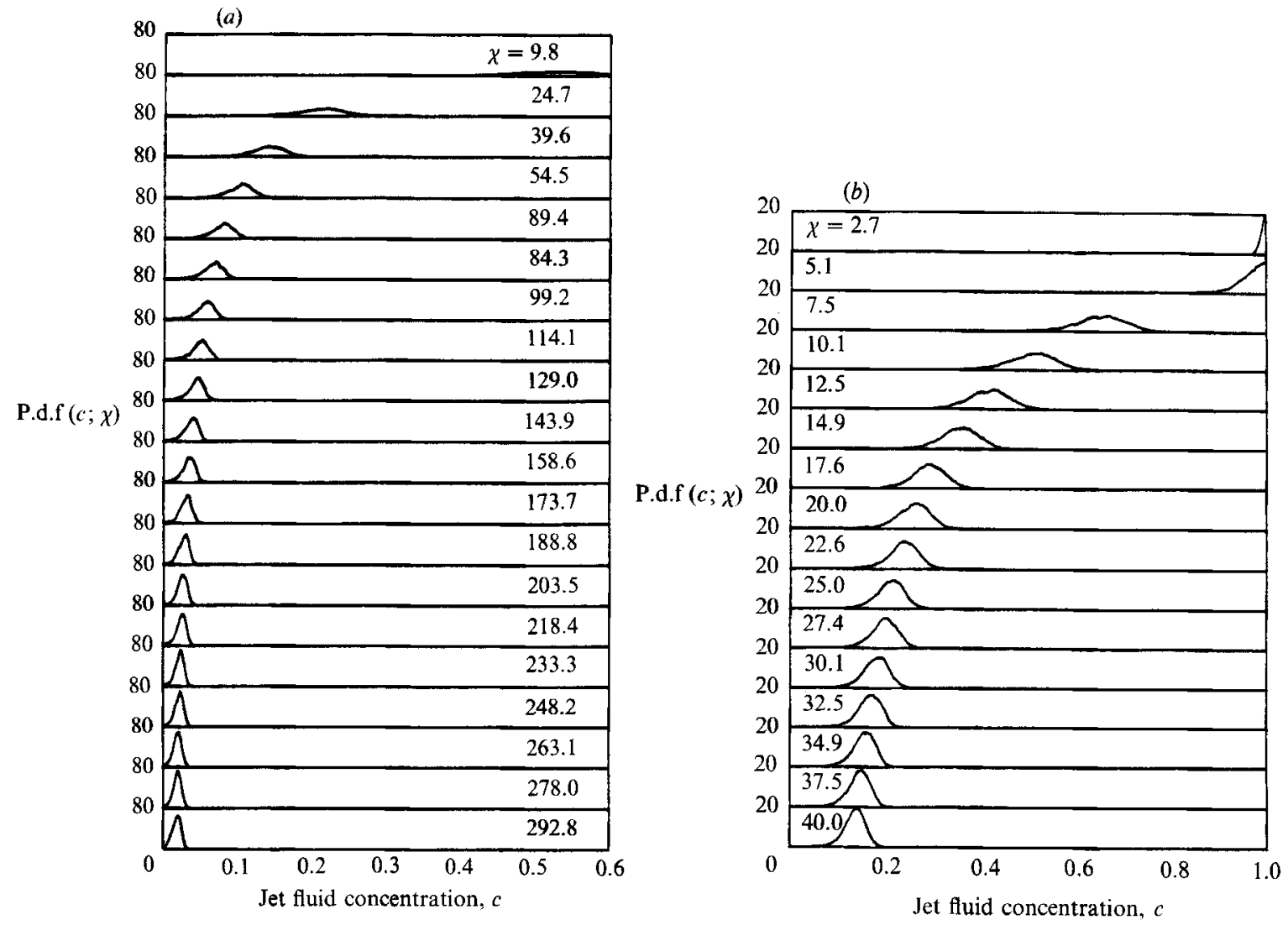

Figure 11. P.d.f.s of the jet fluid concentration $c(\chi, t)$ along the jet centreline at $R e=5000$. (a) $0 \leqslant \chi \leqslant 300,(b) 0 \leqslant \chi \leqslant 40$ detailing the transition from near-field to far-field mixing.

resolution in these measurements was sufficient to resolve nearly all seales in the jet fluid concentration field. Of particular relevance to the discussion in $\$ 5.3$, the radial variation in the amount of unmixed ambient fluid found in the jet can be readily seen in these results. Also evident is the apparent decrease in pure ambient fluid in the jet with increasing Reynolds number.

Similarly, direct measurements of the p.d.f.s at $R e=5000$ obtained from our axial imaging experiments along the jet centreline are shown in figure 11. The results in figure $11(a)$ cover the range $0 \leqslant \chi \leqslant 300$, while the p.d.f.s over the range $0 \leqslant \chi \leqslant 40$ in figure $11(b)$ detail the transition from the near field to self-similar mixing in the far field. Note that while it is generally presumed that the scaled concentration $c / \bar{c}$ is an appropriate similarity concentration variable in the jet far field, and that p.d.f.s of this scaled concentration would be self-similar, a direct check on this selfsimilarity in the p.d.f. is not possible from the present measurements owing to the inherently varying relative resolution with increasing downstream distance (see $\$ 4.1$ ). In the following section, we present a method for indirectly determining the self-similar form of the p.d.f. with effectively molecular resolution.

\subsection{A chemical reaction method for measuring the similarity p.d.f.}

In this section, we use the notion of an appropriately scaled concentration variable in which the p.d.f.s are self-similar, together with the relationship between the p.d.f.s 
(a)

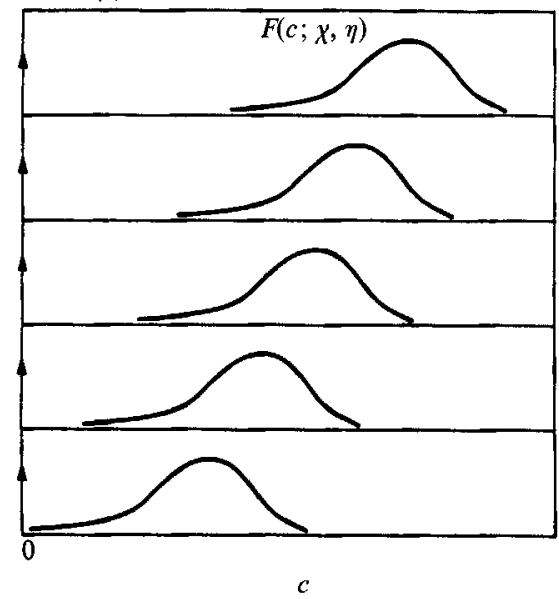

(c)

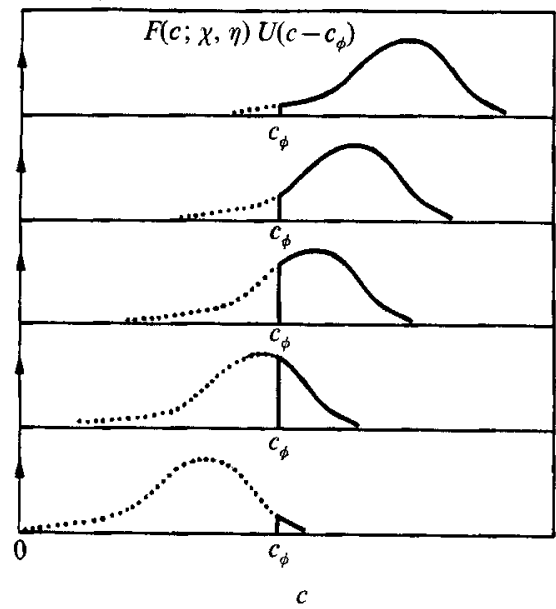

(b)

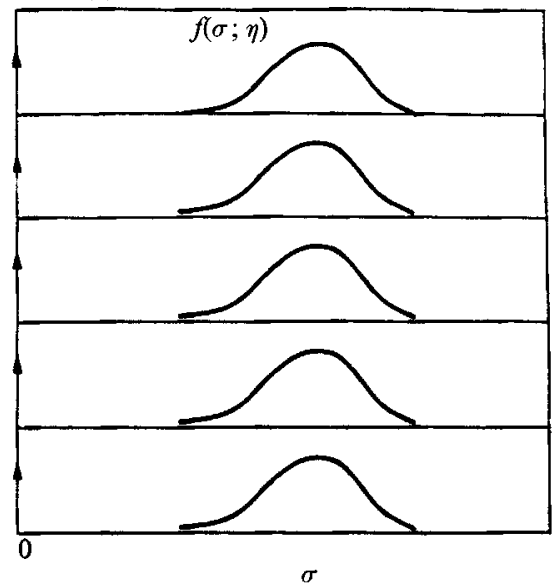

(d)

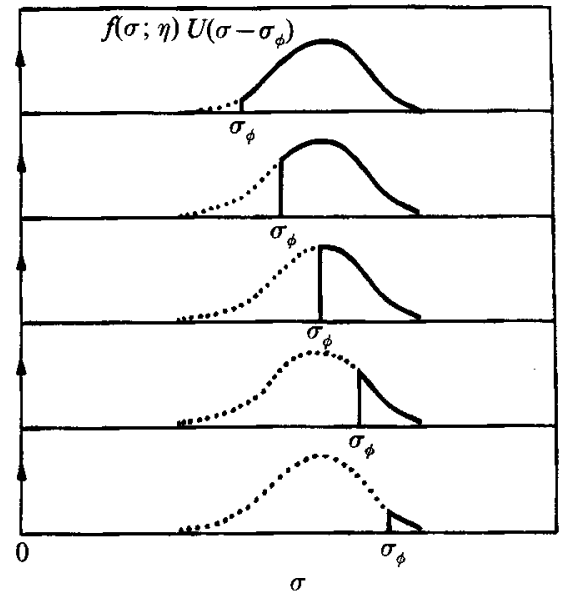

Figure 12. Schematic representation showing p.d.f.s of the scalar concentration $c(\chi, t)$ and the similarity concentration $\sigma(\chi, t)$ for conserved and reactive scalars, on which the chemical reaction method in $\$ 5.5$ is based. This method allows the self-similar form of the p.d.f. in the jet far field to be measured with potentially molecular resolution. $(a)$ P.d.f. $(c ; \chi, \eta)$ in $(4)$ for a conserved scalar. (b) P.d.f. $(\sigma ; \eta)$ in (5) for a conserved scalar. (c) P.d.f. $(c ; \chi, \eta)$ in $(6)$ for a reactive scalar. $(d)$ P.d.f. $(\sigma ; \eta)$ in $(7)$ for a reactive scalar.

for conserved and reactive sealars, to develop a method for measuring the self-similar form of the conserved scalar concentration p.d.f. without the compromising resolution inherent in any direct measurement. The method exploits the sensitivity of a reactive scalar at the molecular scale to yield the p.d.f. with effectively molecular resolution. This same feature of the fluorescein dye was used by Koochesfahani \& Dimotakis (1986) to obtain the mean mixed fluid concentration in the shear layer. However, here we make additional use of the similarity properties of the jet to obtain the concentration p.d.f. directly. The resulting method requires only a measurement of the mean profile of the reactive scalar concentration, which can be readily obtained without resolution error.

As shown schematically in figure $12(a)$, the conserved scalar concentration p.d.f. 
at any point $(\chi, \eta)$ in the far field is composed of a mixed fluid part, denoted as $F(c ; \chi, \eta)$, and a part corresponding to the probability of finding unmixed ambient fluid at that point, denoted as $\bar{p}_{0}(\chi, \eta) \delta(c)$, where $\delta(c)$ is a Dirac delta function, so that

$$
\text { p.d.f. }(c ; \chi, \eta)=F(c ; \chi, \eta)+\bar{p}_{0}(\chi, \eta) \delta(\eta) \text {. }
$$

In terms of a similarity concentration variable $\sigma$, with ambient fluid corresponding to $\sigma=0$, the p.d.f. will be self-similar along any ray $\eta$ and can also be separated into its mixed and unmixed fluid parts, as shown schematically in figure $12(b)$, namely

$$
\text { p.d.f. }(\sigma ; \eta)=f(\sigma ; \eta)+\bar{p}_{0}(\eta) \delta(\sigma) \text {, }
$$

where $f(\sigma ; \eta)$ is the self-similar form of the mixed fluid part of the p.d.f.

Next, we consider how the concentration p.d.f. for a reactive scalar is related to (4), as shown schematically in figure $12(c)$. Denoting the stoichiometric concentration for the fast, reversible reaction as $c_{\phi} \equiv 1 /(1+\phi)$, the previous mixed fluid part $F(c ; \chi, \eta)$ will now be truncated for $c<c_{\phi}$, and the previous unmixed ambient fluid part $\bar{p}_{0}(\eta) \delta(c)$ will be augmented by the mixed fluid at concentrations below $c_{\phi}$, so that

$$
\text { p.d.f. }(c ; \chi, \eta)=F(c ; \chi, \eta) U\left(c-c_{\phi}\right)+\left[\bar{p}_{0}(\eta)+\int_{0^{+}}^{c_{\phi}} F(c ; \chi, \eta) \mathrm{d} c\right] \delta(c),
$$

where $U\left(c-c_{\phi}\right)$ is a unit step function at $c=c_{\phi}$. In terms of the self-similar concentration variable $\sigma$, the stoichiometric concentration is denoted by $\sigma_{\phi}$, which must increase with $\chi$ in order for the reactive scalar to eventually be completely consumed. As a result, the p.d.f.s in terms of $\sigma$ must depend parametrically on $\sigma_{\phi}$ as shown schematically in figure $12(d)$, namely

$$
\text { p.d.f. }\left(\sigma ; \sigma_{\phi}, \eta\right)=f(\sigma ; \eta) U\left(\sigma-\sigma_{\phi}\right)+\left[\bar{p}_{0}(\eta)+\int_{0^{+}}^{\sigma_{\phi}} f(\sigma ; \eta) \mathrm{d} \sigma\right] \delta(\sigma) \text {. }
$$

Although the resolution limitations inherent in any direct concentration measurement preclude the exact determination of $c$ or $\sigma$, the linear averaging over a small region of space and time which is ultimately responsible for this limitation allows an exact measurement of the mean concentration $\bar{c}$ or $\bar{\sigma}$. For any $\sigma_{\phi}$ in (7), the corresponding local mean value of the reactive scalar concentration, $\bar{\sigma}\left(\sigma_{\phi} ; \eta\right)$, is related to the local similarity p.d.f. by

$$
\bar{\sigma}\left(\sigma_{\phi} ; \eta\right)=\int_{0}^{\infty} \sigma \text { p.d.f. }\left(\sigma ; \sigma_{\phi}, \eta\right) \mathrm{d} \sigma
$$

Equation (7) then gives the local mean concentration of the reactive scalar as

$$
\vec{\sigma}\left(\sigma_{\phi} ; \eta\right)=\int_{\sigma_{\phi}}^{\infty} \sigma f(\sigma ; \eta) \mathrm{d} \sigma
$$

The mixed fluid part $f(\sigma ; \eta)$ of the similarity p.d.f. can therefore be determined by differentiating $\bar{\sigma}\left(\sigma_{\phi} ; \eta\right)$ with respect to $\sigma_{\phi}$ to give

$$
f\left(\sigma_{\phi} ; \eta\right)=-\frac{1}{\sigma_{\phi}} \frac{\mathrm{d}}{\mathrm{d} \sigma_{\phi}} \bar{\sigma}\left(\sigma_{\phi} ; \eta\right) .
$$

The remaining unmixed ambient fluid part of the p.d.f. can then be determined from the normalization condition to give

$$
\bar{p}_{0}(\eta)=1-\int_{0^{+}}^{\infty} f(\sigma ; \eta) \mathrm{d} \sigma
$$



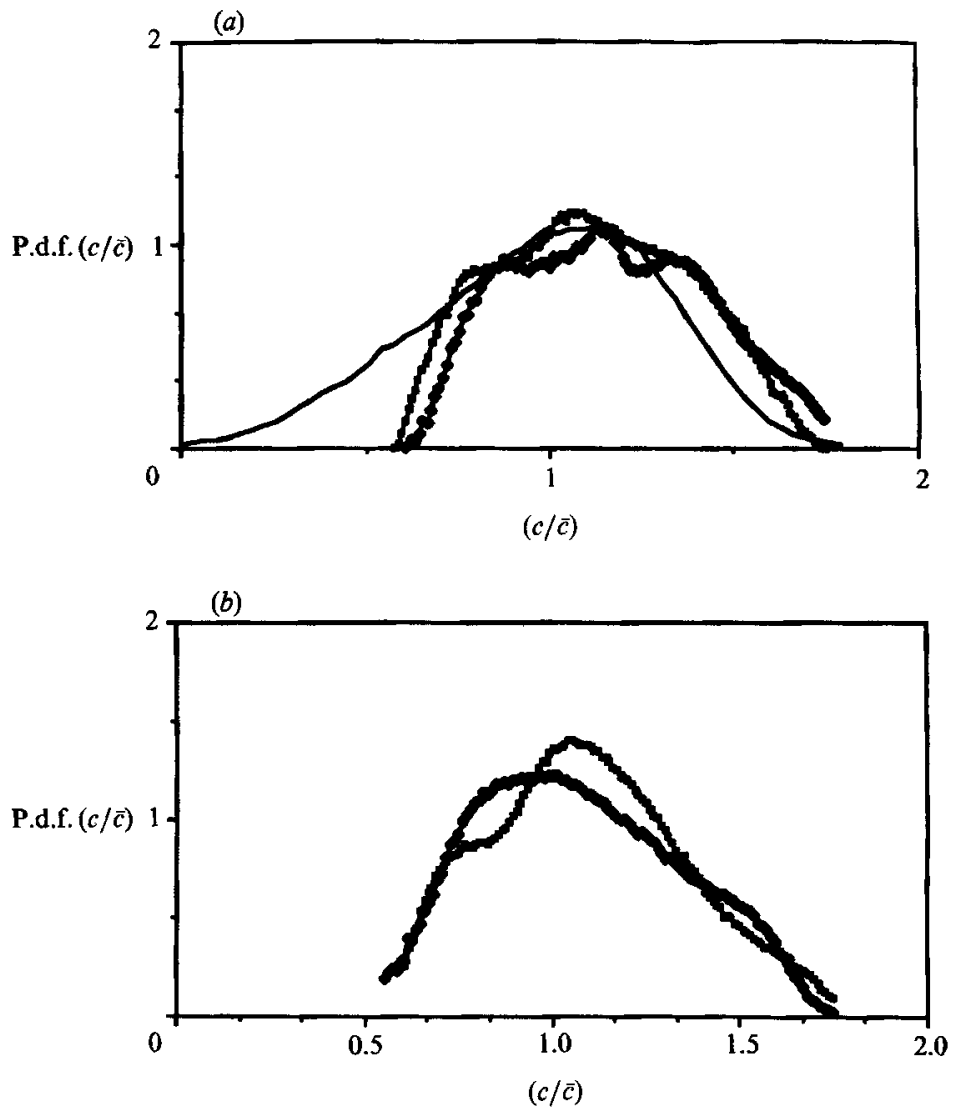

Frgure 13. Result for the self-similar p.d.f. of a conserved scalar on the jet centreline $(\eta=0)$ in the far field obtained from the chemical reaction method in $\$ 5.5$. Notice that the shape of the p.d.f. appears to change as the Reynolds number is increased from 5000 to 20000 . (a) $R e=5000 ;-$, $\phi=23.5 ; \longrightarrow, \phi=28.0 ; \longrightarrow$, from the direct measurement in figure $10(b) .(b) R e=20000 ;-<$, $\phi=23.5 ;-, \phi=26.5$.

Equations (10) and (11) permit the self-similar form of the conserved scalar concentration p.d.f. on any ray $\eta$ to be determined, without resolution limitations, from a single measurement of the mean reactive scalar concentration profile $\vec{\sigma}\left(\sigma_{\phi} ; \eta\right)$ along the ray.

Using this technique with the presumed self-similar concentration variable $\sigma \equiv c / \bar{c}$, the conserved scalar concentration p.d.f. on the jet centreline was measured with $\phi$ ranging from 23.5 to 28.0 (see $\$ 2$ ). Note that if the self-similarity in the p.d.f. on which this method is based were valid for all $\chi>0$, then the similarity p.d.f. could be determined for all $\sigma \geqslant 0$. However, the discussion in $\$ 5.6$ suggests that full similarity in the mean centreline concentration is not attained until $\chi \approx 20$ (see figure $15 b)$, so with $c$ from $(3), f(\sigma ; \eta)$ in $(10)$ can in principle only be determined for $\sigma>3.7 /(1+\phi)$. In practice, $f(\sigma ; \eta)$ could only be determined for $\sigma>0.5$ using this method, with this limitation presumably resulting from the sensitivity of the method to the asymptotic approach of the full p.d.f. to strict self-similarity.

Figure 13 shows the similarity p.d.f.s obtained using this chemical reaction method at Reynolds numbers of 5000 and 20000 , in each case showing results from two separate measurements. While the general form of the p.d.f. obtained from the 
two measurements at each Reynolds number appear to be roughly the same, there are some differences that may be attributable to remaining beam refraction difficulties in the measurements (see $§ 2)$. Also shown for comparison in figure $13(a)$ is the p.d.f. obtained by expressing the direct measurement in figure $10(b)$ at $\eta=0$ in terms of $c / \bar{c}$, where it can be seen that the differences between the results from the direct measurement and the chemical reaction method are qualitatively as would be expected from resolution limitations in the direct measurement.

Two aspects of the p.d.f.s in figure 13 are of particular relevance to this investigation of jet mixing. The first concerns the probability $\bar{p}_{0}(0)$ of finding unmixed ambient fluid on the jet centreline at increasing Reynolds numbers. Following (11), the area under the similarity p.d.f.s in figure 13 below the smallest value of $\sigma$ at which the construction was possible, as discussed above, was found to be 0.17 at $R e=5000$ and 0.08 at $R e=20000$. In view of the inability to construct the similarity p.d.f. for smaller values of $\sigma$, these results should be considered as upper bounds on $\bar{p}_{0}(0)$. They do, however, exceed the values obtained from the direct measurements in $\$ 5.3$, as would be expected. More important, and regardless of their absolute values, they confirm the trend towards decreasing amounts of unmixed ambient fluid reaching the jet centreline with increasing Reynolds number suggested in \$5.3. Secondly, a comparison between the p.d.f.s in figures $13(a)$ and $13(b)$ indicates that, as the Reynolds number is increased from 5000 to 20000 , the overall shape of the p.d.f. changes, reflecting a change in the distribution of mixed fluid compositions in the jet. However, note that the highest concentrations in the p.d.f. remain essentially unchanged, consistent with the Reynolds number independence of the flame length of chemically reacting turbulent jets for $R e>3000$ (Dahm et al. 1984). We can conclude from these p.d.f.s that at least some aspects of the transport and mixing processes in the jet far field are still evolving with increasing Reynolds number between 5000 and 20000 .

\section{6. $\chi$-t reconstructions of the concentration field}

Although each of the individual measured concentration profiles can be displayed separately as in figure 3 , a more direct means for interpreting the time-varying concentration field which these data represent is shown in figure 14 (plate 1). These are $\chi t$ reconstructions of the jet fluid concentration field, shown in terms of a scaled concentration variable $\psi$ defined as

$$
\psi \equiv \chi \frac{c}{1-c}
$$

Pure ambient fluid is thus denoted by $\psi=0$, while pure jet fluid corresponds to $\psi \rightarrow \infty$. For the same data for which the p.d.f.s of $c$ are shown in figure 11, p.d.f.s of this scaled concentration $\psi$ are shown in figure 15. Note that, in terms of $\psi$, the p.d.f.s approach a nearly self-similar form for values of $\chi$ beyond about 20 , this limit presumably denoting the axial distance required for the fully developed self-similar far field of the jet to be reached. Thus, for the purposes of interpreting data from these measurements, this scaled concentration variable demonstrates the necessary self-similarity for comparing axial concentration profiles in figure 14 . It must be emphasized that the scaled concentration $\psi$ is introduced in these measurements only to remove some of the effects of the inherently varying resolution to aid the interpretation of the data.

Figure 14 shows the value of $\psi$ from every second element of the 1024-element array for 500 successively measured axial concentration profiles. The 256 different 

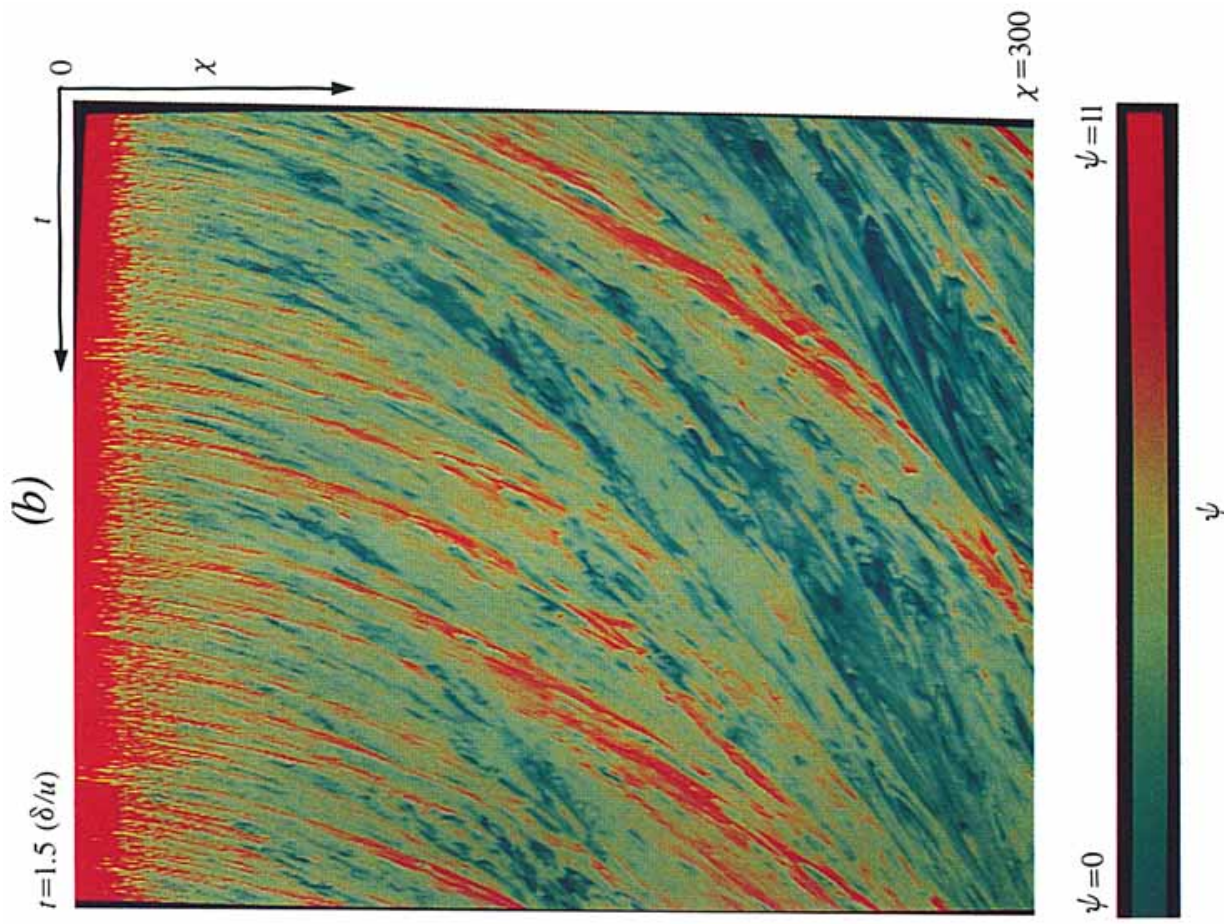

离

要

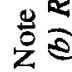

:

ํㅡㄴ

造

ङ

过

造

焉 흠

늘

全

E

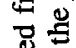

.$\Xi$

뀽. 임
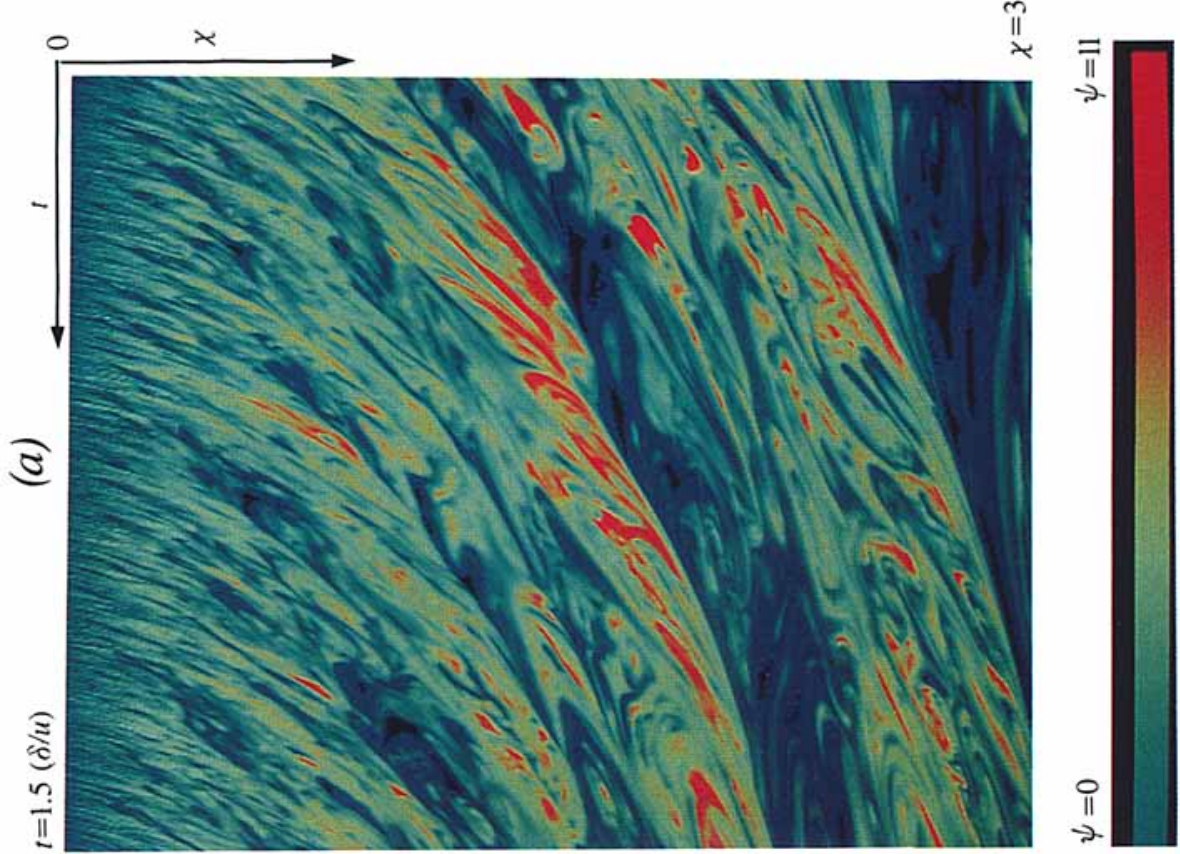

$\rightarrow$ 肴

을

氙

용

迡客

उु

్ㅠㅇ

․ㅗㄴ

它

ㅎํㅇ

5ี

$\rightarrow \quad$ 总

声

通产

迶

$\div$

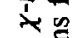

ذ广.

w.

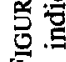
章 
(a)
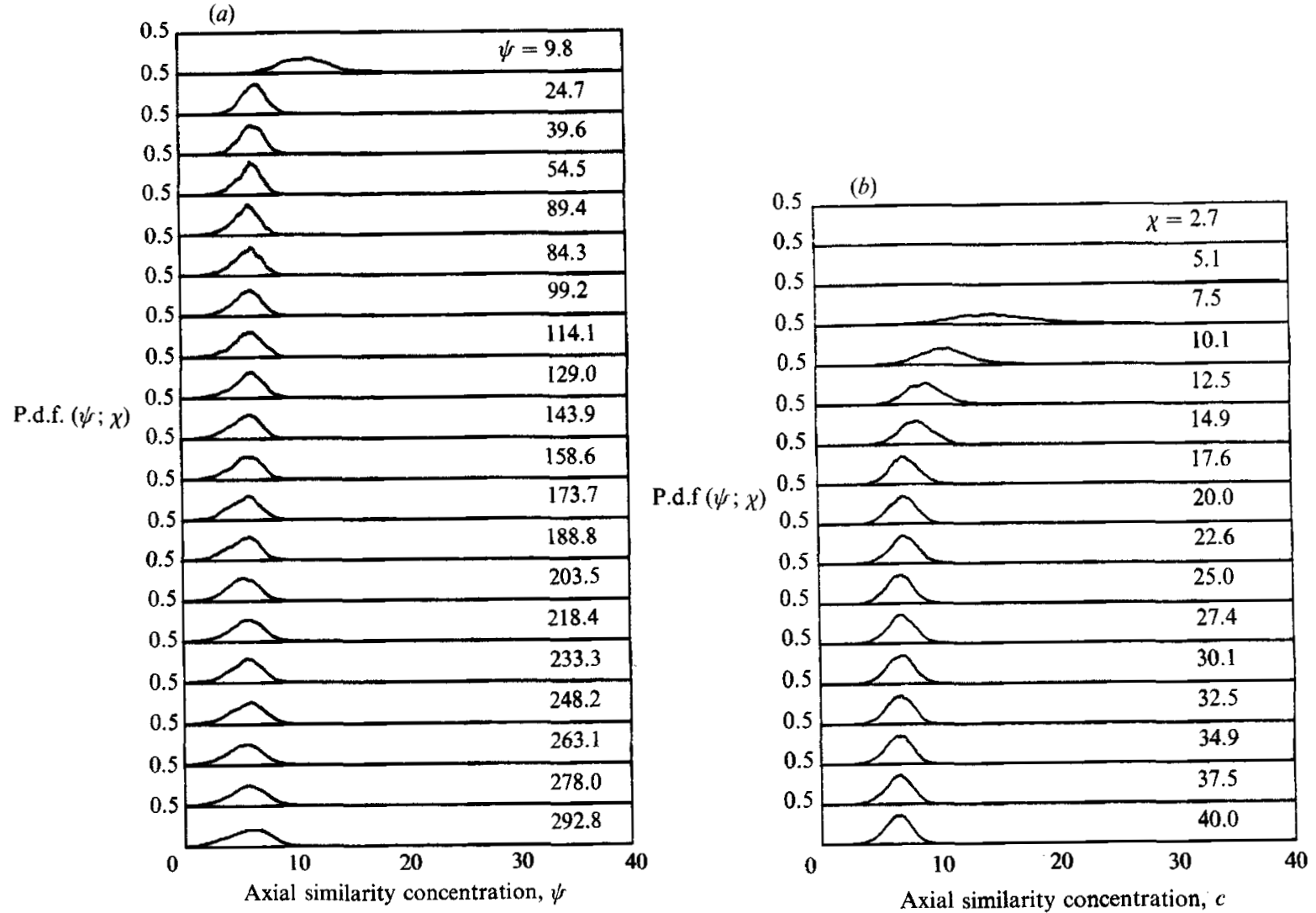

Figure 15. P.d.f.s of the scaled jet fluid concentration $\psi$ in (12) along the centreline at $R e=5000$. Note that, in terms of $\psi$, the p.d.f.s become nearly self-similar in the jet far field, indicating that this scaled concentration effectively removes much of the inherently varying resolution along the jet centreline for the reconstructions in figure 14. (a) $0 \leqslant \chi \leqslant 300,(b) 0 \leqslant \chi \leqslant 40$.

colours were assigned to denote the various scaled concentration levels, with pure red denoting $\psi=11$ and decreasing uniformly to pure blue, with pure black corresponding to unmixed ambient fluid, namely $\psi=0$. Pure green denotes mixed fluid at the local mean scaled concentration $\bar{\psi}=\mathbf{5 . 4}$ obtained from the p.d.f.s in figure 15. The principal feature of this colour assignment is that, owing to the similarity demonstrated in this scaled concentration variable $\psi$, the physical interpretation of these colours is the same everywhere in the jet far field. Consequently, any blue shading denotes mixed fluid at an unusually low concentration for its axial location, while red shadings denote mixed fluid at exceptionally high concentrations for that location. (Note that the characteristic unsteadiness in the breakpoint of the jet at low Reynolds numbers gives a timevarying definition for $\chi=0$ at $R e=1500$. To avoid this complication, $\chi=0$ was taken slightly beyond the longest breakpoint observed. The absence of pure red at small values of $\chi$ in figure $14(a)$, and the deficiency of red in the far field in comparison with figure $14(b)$, is simply an artifact of this choice.) The data in figure 14 cover the range $0<\chi<300$ in the axial direction, and at the farthest downstream location span approximately 1.5 local large-scale times $\delta / u$ in the temporal direction. Each such $\chi^{-t}$ reconstruction consists of more than a quarter of a million individual concentration measurements. 
The data in figure 14 show further evidence for the organization of transport and mixing in the jet far field noted in $\$ 3$, and provide additional details about the structure and dynamics of this organization. First, we note that at any fixed time (i.e. along horizontal lines in these $\chi-t$ diagrams) axial segments are discernible within which the mixed fluid concentration shows a tendency toward red shades near the downstream end and blue shades near the upstream end. As noted above, in terms of $\psi$ such a colour pattern indicates a region within which the mixed fluid concentration is approximately uniform. Furthermore, the axial length of the regions within which this colour pattern is found can be seen to increase with downstream distance. Indeed the length of these regions in figure 14 is typically about half their downstream location, consistent with their size being approximately equal to the full local width of the flow $(\delta \approx 0.44 x)$. Moreover, the time required for these regions to pass any fixed point (i.e. their size in the temporal direction in these $\chi-t$ diagrams) increases roughly quadratically with $\mathcal{X}$, as evidenced by their typically parabolic trajectories in these diagrams. These trajectories indicate that the speed at which the regions progress downstream decreases approximately as $\chi^{-1}$, consistent with the similarity scaling demanded of the mean velocity field. Data such as those in figure 14 also give some indication of the dynamics of these regions. Interactions can be discerned in which initially distinct adjacent regions undergo some form of coalescence to form a new, larger region; however, the details of how this interaction proceeds are not easily discernible. Nevertheless, it can be seen that these regions typically persist for a duration close to the local large-scale time of the flow, which increases quadratically with $\chi$, before coalescing with an upstream or downstream neighbouring region. Qualitative evidence of such interactions can also be seen in motion films of flow visualizations of the type shown in figure 1.

\section{Discussion and conclusions}

One of the objectives of this investigation has been to help clarify the nature of large-scale organization of mixing in the far field of turbulent jets. To this end, the flow visualizations in $\S 3$ and results from the imaging measurements in $\$ 5$ have given direct evidence for the presence of such an organization. Collectively, they verify that the instantaneous concentration field of the jet consists of an ordered sequence of regions, typically spanning an axial and lateral scale the size of the local width of the flow, within which the composition of the mixed fluid shows no systematic lateral gradient and only a very weak axial gradient. These results largely confirm the simple conceptual picture of organization in the jet proposed in [I] on the basis of the flame length fluctuations of chemically reacting jets, though some indications of a spiral structure have also been observed here as well as in the flow visualizations of Dimotakis et al. (1983b) and the flame length fluctuations of Mungal \& O'Neil (1989). Moreover, the flame length fluctuations themselves show that this organization in the jet plays a crucial role for transport and mixing of the jet and ambient fluids.

We note that this organization of the jet far field bears some similarities to that in fully developed turbulent shear layers, though there are many important differences. For example, the transport of unmixed ambient fluid deep into the region of shear is common to both flows, though our results indicate a Reynolds number dependence that has not been observed in shear layers. In the shear layer, the role of large spanwise vortical structures in such transport can be considered as largely understood, and suggests some form of large vortical structures in the jet far field. In addition, the uniformity of the mixed fluid composition within large regions is also 
a common feature of both flows. The role of the large spanwise vortices in preventing a systematic variation in the mixed fluid composition in turbulent shear layers lends further support to the notion of large vortical structures in the far field of turbulent jets. However, the nature of such structures in the jet and interactions among them are apparently quite different from the shear layer. The organization in the jet far field appears to be both topologically and dynamically more complex than that in turbulent shear layers, and may be a consequence of the fact that the jet is simultaneously unstable both to axisymmetric and helical modes, and appears to alternate between these two.

These results have also established the proper form of the similarity scaling for the mean conserved scalar concentration in the jet far field, and give the scaling constant as 5.4. They further suggest that the level of concentration fluctuations in the jet far field is approximately $\mathbf{0 . 2 2 5}$. Our results directly show the presence of pure ambient fluid on the jet centreline, whereas high-resolution measurements in the self-similar far field of $S c \approx 1$ turbulent jets show no such unmixed fluid at comparable Reynolds numbers, indicating an effect of the Schmidt number on mixing in turbulent jets. In particular, our large- $S c$ data show that pure ambient fluid penetrates well into the jet, occupying as much as $30 \%$ of the jet centreline at $R e=1500$ but decreasing with increasing Reynolds number even when resolution effects are taken into account. Furthermore, the repetitive large-scale ramp-like features evident in conserved scalar time traces in gaseous jet mixing do not consistently appear in our data at large $S c$ at the Reynolds numbers investigated, suggesting a further possible effect of the molecular diffusivity on mixing in the jet. Here again the similarity with the shear layer is noteworthy, as pointed out in $\$ 5.3$.

The chemical reaction method used for measuring the p.d.f.s with potentially molecular resolution provide further data for comparison with similar measurements in gaseous jets. In particular, the distribution of mixed fluid compositions in the jet also appears to change with increasing Reynolds number, as evidenced by the apparent change in shape of the conserved scalar concentration p.d.f. between Reynolds numbers 5000 and 20000 , indicating that some aspects of mixing in the jet far field have not yet become Reynolds number independent. However, the highest concentrations in the p.d.f. do not change over this range of Reynolds numbers.

We would like to acknowledge valuable contributions by Dr M. M. Koochesfahani in the development of the imaging techniques, and numerous discussions with Dr J. E. Broadwell. This work was sponsored, in part, by the Gas Research Institute under Grant No. 5083-260-0878, by the Air Force Office of Scientific Research under Grant No. 83-0213 and Contract No. F49620-79-0159, and by the Energy and Environmental Research Corporation under Contract No. 8400-28.

\section{Appendix}

Comparisons with the mean jet fluid concentration scaling along the centreline are used in these experiments as a partial validation of the flow, the concentration imaging technique, and the data reduction technique. While the $x^{-1}$ functional form of this scaling is well known, its proper dimensionless representation does not appear to be widely understood and has been a source of confusion in the interpretation of measurements in turbulent jets. Moreover, the proportionality constant in this scaling law has not been well established; in a review of data from several different experiments, Chen $\&$ Rodi (1980) suggest an approximate value of about 5 . In the 
following analysis, an integral mass balance and self-similarity in radial profiles for the mean and fluctuating quantities are used to obtain a more accurate estimate for this proportionality constant. The analysis also yields the proper dimensionless form of the mean concentration sealing in the far field of axisymmetric turbulent jets.

\section{A.1. The far field equivalent source diameter}

At axial distances for which the local jet mass flux, $\dot{m}(x)$, defined as

$$
\dot{m}(x)=\int_{0}^{\infty} \overline{\rho(x, r) u(x, r)} 2 \pi r \mathrm{~d} r,
$$

is sufficiently large compared with its source mass flux $\dot{m}_{0}$, the flow is generally presumed to be characterized entirely by the jet source momentum flux $J_{0}$ and the ambient fluid density $\rho_{\infty}$. Dimensional reasoning then suggests that the jet mass flux should scale as

$$
\dot{m}(x) \sim\left(\rho_{\infty} J_{0}\right)^{\frac{1}{2}} x .
$$

Measurements by Ricou \& Spalding (1961) have verified this functional form and give the proportionality constant in (A 2) as 0.282 . (Alternatively, integrating the mean velocity profile of Wygnanski \& Fiedler 1969 gives this constant as 0.244. Ricou \& Spalding discuss the difficulties associated with such integral estimates of this constant, and we assume their value to be the more accurate one.) From an integral mass balance, the jet fluid concentration, density and velocity fields are related to the source mass flux as

$$
\int_{0}^{\infty} \overline{c(x, r) \rho(x, r) u(x, r)} 2 \pi r \mathrm{~d} r=\dot{m}_{0} .
$$

Together with (A 1) and (A 2), this gives

$$
\frac{\int_{0}^{\infty} \overline{c(x, r) \rho(x, r) u(x, r)} 2 \pi r \mathrm{~d} r}{\int_{0}^{\infty} \overline{\rho(x, r) u(x, r)} 2 \pi r \mathrm{~d} r}=3.14 \frac{2 \dot{m}_{0}}{\left(\pi \rho_{\infty} J_{0}\right)^{\frac{1}{2}}} x^{-1} .
$$

The grouping on the right-hand side of (A 4) suggests the proper dimensionless form for the axial coordinate of the jet fluid concentration in the far field. We define the 'far field equivalent source diameter' $d^{*}$ as

$$
d^{*} \equiv \frac{2 \dot{m}_{0}}{\left(\pi \rho_{\infty} J_{0}\right)^{\frac{1}{2}}}
$$

and recognize the dimensionless axial coordinate in (A 4) as

$$
\chi \equiv\left(x / d^{*}\right) \text {. }
$$

Physically, $d^{*}$ is the diameter through which fluid at density $\rho_{\infty}$ and pressure $p_{\infty}$ would flow with the same mass flux $\dot{m}_{0}$ and momentum flux $J_{0}$ as the actual source. Note that if the jet source is circular and has uniform exit profiles of density and velocity, then $d^{*}=\left(\rho_{0} / \rho_{\infty}\right)^{\frac{1}{2}} d_{0}$, with $d_{0}$ the physical diameter of the jet source. This is the familiar, but more restrictive, form of the far field equivalent source diameter. The general form in (A 5) was first introduced by Thring \& Newby (1952) to correlate enclosed turbulent jet diffusion flame lengths, and has been used in a similar connection by Avery \& Faeth (1974), Becker \& Liang (1978) and Becker \& Yamazaki (1978). However, the arguments leading to (A 4) indicate that $d^{*}$ plays the broader role of properly normalizing the downstream distance in the scaling for the jet fluid 
concentration in the far field of axisymmetric turbulent jets. The correlation of flame lengths with $d^{*}$ then follows directly from this, as indicated in figure 1 of [I].

\section{A.2. The mean centreline concentration scaling}

The concentration, density and velocity fields appearing in (A 4) can be expressed in terms of their conventional temporal mean and fluctuating constituents as

$$
\begin{aligned}
& c(x, r)=\bar{c}(x, r)+c^{\prime}(x, r), \\
& \rho(x, r)=\rho_{\infty}+\overline{\Delta \rho}(x, r)+\Delta \rho^{\prime}(x, r), \\
& u(x, r)=\bar{u}(x, r)+u^{\prime}(x, r) .
\end{aligned}
$$

If radial profiles of the mean and fluctuation correlations, which arise when (A $6 a-c)$ are introduced in (A 4), follow similarity scalings of the form

$$
\begin{aligned}
\bar{c}(x, r) & =\bar{c}(x) f(\eta), \\
\overline{\Delta \rho}(x, r) & =\overline{\Delta \rho}(x) g(\eta), \\
\bar{u}(x, r) & =\bar{u}(x) h(\eta), \\
\overline{c^{\prime} \Delta \rho^{\prime}}(x, r) & =\bar{c}(x) \overline{\Delta \rho}(x) i(\eta), \\
\overline{\Delta \rho^{\prime} u^{\prime}}(x, r) & =\overline{\Delta \rho}(x) \bar{u}(x) j(\eta), \\
\overline{c^{\prime} u^{\prime}}(x, r) & =\bar{c}(x) \bar{u}(x) k(\eta), \\
\overline{c^{\prime} \Delta \rho^{\prime} u^{\prime}}(x, r) & =\bar{c}(x) \overline{\Delta \rho}(x) \bar{u}(x) l(\eta),
\end{aligned}
$$

with

$$
\eta \equiv r / x,
$$

then the scaling law for the mean concentration along the centreline is obtained as

$$
\bar{c}(x)=\frac{3.14}{\Lambda(\chi)} \chi^{-1},
$$

where

$$
\Lambda(\chi) \equiv \frac{I_{1}+\frac{\overline{\Delta \rho}(\chi)}{\rho_{\infty}} I_{2}}{I_{3}+\frac{\overline{\Delta \rho}(\chi)}{\rho_{\infty}} I_{4}}
$$

and where $I_{1} \ldots I_{4}$ are integral invariants, given by

$$
\begin{aligned}
I_{1} & \equiv \int_{0}^{\infty}[f(\eta) h(\eta)+k(\eta)] \eta \mathrm{d} \eta \\
I_{2} & =\int_{0}^{\infty}[f(\eta) g(\eta) h(\eta)+h(\eta) i(\eta)+f(\eta) j(\eta)+g(\eta) k(\eta)+l(\eta)] \eta \mathrm{d} \eta \\
I_{3} & =\int_{0}^{\infty} h(\eta) \eta \mathrm{d} \eta \\
I_{4} & =\int_{0}^{\infty}[g(\eta) h(\eta)+j(\eta)] \eta \mathrm{d} \eta
\end{aligned}
$$

If the densities are matched, or at axial distances for which any initial density difference has become negligible, then

$$
\Lambda(\chi)=I_{1} / I_{3} \equiv \lambda
$$


giving the scaling law for the mean concentration along the centreline as

$$
\bar{c}(x)=\frac{3.14}{\lambda} \chi^{-1} .
$$

The value of the scaling constant in (A 12) can be determined from (A 11) and (A $10 a, c)$ if the profiles $f(\eta), h(\eta)$ and $k(\eta)$ are known. Accurate measurements of the $\overline{u^{\prime} c^{\prime}}$ profile for the jet do not appear in the literature, however $k(\eta)$ can be approximated from the measured profile $\overline{u^{\prime} \zeta^{\prime}}$ of Dibble, Kollmann \& Schefer (1984) near the jet-like limit (small $x / \vartheta$ ) of an axisymmetric coflowing turbulent jet (see Dahm \& Dibble 1988). With the profile $f(\eta)$ given in figure $5(a)$ of $[\mathrm{I}]$ and the profile $h(\eta)$ given by Wygnanski \& Fiedler (1969), a simple quadrature gives $\lambda \approx 0.610$. Equation (A 12) then gives the complete scaling law for the mean centreline jet fluid concentration in the far field as

$$
\bar{c}(x) \approx 5.15 \chi^{-1} .
$$

The principal features of (A 13) are the properly normalized form of the scaling law and the numerical evaluation of the scaling constant from integrals of measured similarity profiles. The value in (A 13) can be compared with that obtained experimentally in figures 4 and 5 from the axial concentration profile measurements in $\$ 5$. Note that, in view of the approximate nature of the $k(\eta)$ profile used, if its contribution in (A 10a) is ignored then an upper bound for the scaling constant in (A 13) is obtained as 5.70 .

\section{REFERENCES}

Antonia, R. A., Prabhu, A. \& Stephenson, S. E. 1975 Conditionally sampled measurements in a heated turbulent jet. J. Fluid Mech. 72, 455-480.

Avery, J. F. \& FAETH, G. M. 1974 Combustion of a submerged gaseous oxidizer jet in a liquid metal. In Proc. 15th Intl Symp. on Combustion, pp. 502-512. The Combustion Institute.

Beavers, G. S. \& Wilson, T. A. 1970 Vortex growth in jets. J. Fluid Mech. 44, 97-112.

Becker, H. A., Hottel, H. C. \& Wirliams, G. C. 1967 The nozzle-fluid concentration field of the round turbulent jet. $J$. F'luid Mech. 30, 285-303.

Becker, H. A. \& Liang, D. 1978 Visible lengths of vertical free turbulent diffusion flames. Combust. Flame 33, 115-137.

Becker, H. A. \& Massaro, T. A. 1968 Vortex evolution in a round jet. J. Fluid Mech. 31, $435-448$.

Becker, H. A. \& Yamazaki, S. 1978 Entrainment, momentum flux and temperature in vertical free turbulent diffusion flames. Combust. Flame 33, 123-149.

Birch, A. D., Brown, D. R., Dodson, M. G. \& Thomas, J. R. 1978 The turbulent concentration field of a methane jet. J. Fluid Mech. 88, 431-449.

Bradshaw, P., Ferriss, D. H. \& Johnson, R. F. 1964 Turbulence in the noise-producing region of a circular jet. J. Fluid Mech. 19, 591-624.

Breidenthal, R. E. 1981 Structure of turbulent mixing layers and wakes using a chemical reaction. J. Fluid Mech. 109, 1-24.

BROADWELL, J. E. 1987 A model for reactions in turbulent jets : Effects of Reynolds, Schmidt, and Damköhler numbers. In Proc. US-France Joint Workshop on Turbulent Reactive Flows; also AFOSR Rep. AFOSR-83-0213.

Broadwel.l, J. E. \& Mungal, M. G. 1986 The effects of Damköhler number in a turbulent shear layer. In Proc. 22nd Intl Symp. on Combustion, pp. 579-587. The Combustion Institute.

Brown, G. L. \& Roshro, A. 1971 The effect of density difference on the turbulent mixing layer. In Turbulent Shear Flows. AGARD-CP-93, pp. 23.1-12.

Brown, G. L. \& Roshko, A. 1974 On density effects and large structure in turbulent mixing layers. J. Fluid Mech. 64, 775-816. 
Chen, C. J. \& Rodr, W. 1980 Vertical Turbulent Buoyant Jets: A Review of Experimental Data. Pergamon.

Chevray, R. \& Tutu, N. K. 1978 Intermittency and preferential transport of heat in a round jet. J. Fluid Mech. 88, 133-160.

Crow, S. C. \& Champagne, F. H. 1971 Orderly structure in jet turbulence. J. Fluid Mech. 48, 547-591.

DAHM, W. J. A. 1985 Experiments on entrainment, mixing and chemical reactions in turbulent jets at large Schmidt number. Ph.D. thesis, Caltech.

DaHM, W. J. A. \& BUCH, K. A. 1989 High resolution three-dimensional $\left(256^{3}\right)$ spatio-temporal measurements of the conserved scalar field in turbulent shear flows. In Proc. 7th Symp. on Turb. Shear Flows, vol. 1, pp. 14.1.1-14.1.6; to appear in Turbulent Shear Flows 7. Springer, 1990.

Dahm, W. J. A. \& Dibble, R. W. 1988 Coflowing turbulent jet diffusion flame blowout. In Proc. 22nd Intl Symp. on Combustion, pp. 801-808. The Combustion Institute.

Dанм, W. J. A. \& Dimotakis, P. E. 1987 Measurements of entrainment and mixing in turbulent jets. AIAA J. 25, 1216-1223; also AIAA Paper 85-0056. (Referred to herein as [I].)

Dahm, W. J. A., Dimotakis, P. E. \& Broadwell, J. E. 1984 Nonpremixed turbulent jet flames. AIAA Paper 84-0369.

Dibble, R. W., Kollmax, W. \& Sohefer, R. W. 1984 Conserved scalar fluxes measured in a turbulent nonpremixed flame by combined laser Doppler velocimetry and laser Raman scattering. Combust. Flame 55, 307-321.

Dimotakis, P. E., Broadwell, J. E. \& Howard, R. D. $1983 a$ Chemically reacting turbulent jets. AIAA Paper 83-0474.

Dimotakis, P. E. \& Brown, G. L. 1976 The mixing layer at high Reynolds number: large structure dynamies and entrainment. J. Fluid Mech. 78, 535-560.

Dimotakis, P. E., Miake-Lye, R. C. \& Papantoniod, D. A. $1983 b$ Structure and dynamics of round turbulent jets. Phys. Fluids 26, 3185-3192.

Dowling, D. R. 1988 Mixing in gas phase turbulent jets. Ph.D. thesis, Caltech.

Dowling, D. R. \& Dimotakis, P. E. 1988 On mixing and structure of the concentration field of turbulent jets. Proc. 1st National Fluid Dynamics Congress. New York: AIAA.

Fiedler, H. F. 1974 Transport of heat across a plane turbulent mixing layer. Adv. Geophys. 18 A, 93-109.

Gibson, C. H., Friehe, C. A. \& MoConneld, S. O. 1977 Structure of sheared turbulent fields. Phys. Fluids 20, S156-S167.

HоттеL, H. C. 1953 Burning in laminar and turbulent fuel jets. 4th Intl Symp. on Combustion, pp. 97-113. The Williams and Wilkins Co.

KONRAD, J. H. 1976 An experimental investigation of mixing in two-dimensional turbulent shear flows with application to diffusion-limited chemical reactions. Ph.D. thesis, Caltech; also Project SQUID Tech. Rep. CIT-8-PU.

Koochesfahani, M. M. \& Dimotakis, P. E. 1986 Mixing and chemical reactions in a turbulent mixing layer. J. Fluid Mech. 170, 83-112.

Lockwood, F. C. \& MoneIB, H. A. 1980 Fluctuating temperature measurements in a heated round jet. Combust. Sci. Tech. 22, 63-81.

Mollo-Christensen, E. 1967 Jet noise and shear flow instability seen from an experimenter's viewpoint. Trans. ASME E: J. Appl. Mech. 89, 1-7.

Mungal, M. G. \& Dimotakis, P. E. 1984 Mixing and combustion with low heat release in a turbulent shear layer. J. Fluid Mech. 148, 349-382.

Mungat, M. G. \& Frieler, C. E. 1985 The effects of Damköhler number on a turbulent shear layer - Experimental results. GALCIT' Rep. FM85-01.

Mungal, M. G. \& Hollinasworth, D. K. 1989 Organized motion in a very high Reynolds number jet. Phys. Fluids A 1, 1615-1624.

Muvgal, M. G. \& O'NeIL, J. M. 1989 Visual observations of a turbulent diffusion flame. Combust. and Flame 78, 377-389.

Nakamura, I., Sakai, Y. \& Miyata, M. 1982 A study of the fluctuation concentration field in a turbulent jet. Nagoya Univ. Res. Rep. 34, 113-124. 
Papanicolaov, P. N. \& List, E. J. 1988 Investigations of round vertical turbulent buoyant jets. J. Fluid Mech. 195, 341-391.

Papantoniou, D. A. 1985 Observations of turbulent buoyant jets by use of laser-induced fuorescence. Ph.D. thesis, Caltech.

Papantoniou, D. A. \& List, E. J. 1989 Large scale structure in the far field of buoyant jets. J. Fluid Mech. 209, 151-190.

Pitts, W. M. \& Kashiwagt, T. 1984 The application of Rayleigh light scattering to the study of turbulent mixing. J. Fluid Mech. 141, 391-429.

Ricou, F. P. \& Spaldina, D. B. 1961 Measurements of entrainment by axisymmetrical turbulent jets. J. Fluid Mech. 11, 21-32.

SHLIEN, D. J. 1987 Observations of dispersions of entrained fluid in the self-preserving region of a turbulent jet. $J$. Fluid Mech. 183, 163-173.

Thring, M. W. \& Newby, M. P. 1952 Combustion length of enclosed turbulent jet flames. 4th Intl Symp. on Combustion, pp. 789-796. The Williams and Wilkins Co.

Tso, J., Kovasznay, L. S. G. \& Hussain, A. K. M. F. 1981 Search for large-scale coherent structure in the nearly self-preserving region of a turbulent axisymmetric jet. Trans. ASME I: J. Fluids Engng 103, 503-508.

Wilson, R. A. M. \& Danckwerts, P. V. 1964 Studies in turbulent jet mixing - II. A hot air jet. Chem. Engng Sci. 19, 885-895.

Winant, C. D.\& Browand, F. K. 1974 Vortex pairing: the mechanism of turbulent mixing layer growth at moderate Reynolds number. J. Fluid Mech. 63, 237-255.

Wygnanski, I. \& Fiedler, H. E. 1969 Some measurements in the self-preserving jet. J. Fluid Mech. 38, 577-612.

YUle, A. J. 1973 Large scale structure in the mixing layer of a round jet. J. Fluid Mech. 89, 413-432. 